\title{
Hybrid-Trefftz six-node triangular finite element models for Helmholtz problem
}

\author{
K. Y. Sze • G. H. Liu
}

Received: 8 September 2009 / Accepted: 14 March 2010 / Published online: 4 April 2010

(C) The Author(s) 2010. This article is published with open access at Springerlink.com

\begin{abstract}
In this paper, six-node hybrid-Trefftz triangular finite element models which can readily be incorporated into the standard finite element program framework in the form of additional element subroutines are devised via a hybrid variational principle for Helmholtz problem. In these elements, domain and boundary variables are independently assumed. The former is truncated from the Trefftz solution sets and the latter is obtained by the standard polynomial-based nodal interpolation. The equality of the two variables are enforced along the element boundary. Both the plane-wave solutions and Bessel solutions are employed to construct the domain variable. For full rankness of the element matrix, a minimal of six domain modes are required. By using local coordinates and directions, rank sufficient and invariant elements with six plane-wave modes, six Bessel solution modes and seven Bessel solution modes are devised. Numerical studies indicate that the hybrid-Trefftz elements are typically $50 \%$ less erroneous than their continuous Galerkin element counterpart.
\end{abstract}

Keywords Hybrid-Trefftz - Triangular - Finite element . Helmholtz · Displacement-frame

\section{Introduction}

In the hybrid finite element method for stress/structural analyses, the compatible displacement-based (or, continuous Galerkin) finite element models can be enhanced by introducing stress, strain and/or another displacement as the

\section{K. Y. Sze (更) · G. H. Liu}

Department of Mechanical Engineering,

The University of Hong Kong,

Pokfulam, Hong Kong, People's Republic of China

e-mail: kysze@hku.hk additional field variable(s) to the displacement obtained by polynomial-based nodal interpolation [1-10]. In the hybriddisplacement method, the additional field is a domain displacement $\mathbf{u}_{d}$ which leads to equilibrating stress and may also satisfy some homogeneous boundary conditions [2-4]. This category of hybrid elements are also known as hybridTrefftz or Trefftz elements linked by boundary displacement or displacement-frame [6-9]. The underlying reasons are that the domain displacement is truncated from a Trefftz solution set, which is the basis of various Trefftz methods [11-15], and the boundary or frame variable $\mathbf{u}_{b}$ linking adjacent elements is a displacement. With the displacement compatibility and boundary conditions satisfied by $\mathbf{u}_{b}$ as a priori, the variational functional $\Pi$ of the hybrid-displacement finite element method in the absence of body force can be expressed as [24]:

$$
\begin{array}{r}
\Pi=\sum_{e} \Pi^{e} \text { and } \Pi^{e}=\frac{1}{2} \int_{\Omega^{e}} \boldsymbol{\varepsilon}_{d}^{T} \mathbf{C} \boldsymbol{\varepsilon}_{d} d \Omega \\
-\int_{\partial \Omega^{e}} \mathbf{t}_{d}^{T}\left(\mathbf{u}_{d}-\mathbf{u}_{b}\right) d \Gamma-\int_{S_{t}^{e}} \overline{\mathbf{t}}^{T} \mathbf{u}_{b} d \Gamma
\end{array}
$$

where $\boldsymbol{\varepsilon}_{d}$ and $\mathbf{t}_{d}$ are respectively the strain and traction derived from the domain displacement $\mathbf{u}_{d}, \mathbf{C}$ is the elasticity matrix, $\Omega^{e}$ is the element domain, $\partial \Omega^{e}$ denotes the boundary of $\Omega^{e}, \overline{\mathbf{t}}$ is the prescribed traction over $S_{t}^{e} \subset \partial \Omega^{e}$. If the stress derived from $\mathbf{u}_{d}$ also satisfies the stress equilibrium condition, $\Pi^{e}$ can also be written as:

$\Pi^{e}=\int_{\partial \Omega^{e}}\left(-\frac{1}{2} \mathbf{t}_{d}^{T} \mathbf{u}_{d}+\mathbf{t}_{d}^{T} \mathbf{u}_{b}\right) d \Gamma-\int_{S_{t}^{e}} \overline{\mathbf{t}}^{T} \mathbf{u}_{b} d \Gamma$

which does not involves any domain integration. The analogy of (2) for Helmholtz problems will be employed in this paper. 
A major challenge in the finite element analysis of the Helmholtz problem is that the solution and its derivatives are spatially oscillating in the entire problem domain. While considerable computational saving can be realized by using graded meshes in stress analyses, the practice is not applicable to the Helmholtz problem. The high mesh-density requirement induces tremendous computing load when the wavenumber goes up. To better tackle the issue, a number of wave-based approaches that make use of the solution sets for the wave or Helmholtz equations have been proposed. While a brief survey will be presented in the subsequent paragraphs, the readers may like to consult reference [16] for a more comprehensive review.

In the plane-wave basis method, the plane-wave solutions are employed as the nodal enrichment functions in the context of the partition of unity finite element method [17-21]. The value of the Helmholtz variable at a node is the sum of the plane-wave modes propagating along different directions. Within the element, the Helmholtz variable is obtained by the standard polynomial-based nodal interpolation. Thus, the system equation unknowns are the amplitudes of the planewave modes at the nodes but not the nodal values of the Helmholtz variable.

In the multiscale-based discontinuous enrichment method, the coarse scale approximation constructed by the polynomial-based nodal interpolation is enriched by plane-wave solutions. The enrichment which is intended to resolve the fine scale phenomenon induces discontinuity across the interelement boundary [22,23]. Enforcement of the continuity is conducted through Lagrange multipliers. While the fine scale enrichments can be condensed at element level, the Lagrange multipliers which link the enrichments of adjacent elements enter the system equation.

Following the success of the Trefftz boundary element methods for Helmholtz problems [11-16], a number of Trefftz finite element methods can be noted in literature. A core issue of the Trefftz finite element methods is the enforcement of inter-element continuity of the domain or intra-element variable. The two major categories of Trefftz finite element methods are the hybrid-Trefftz and frameless Trefftz methods.

In the hybrid-Trefftz methods, a boundary or frame variable is introduced in addition to the domain variable. Interelement continuity of the domain variables is indirectly enforced by imposing equality constraints in each of which the equality of the domain variable in an element is constrained (mostly, in a collocation or a weak sense) to the boundary variable defined along the inter-element boundary. Hence, the domain variable can be condensed at the element level. To date, most hybrid-Trefftz finite element methods for Helmholtz problems are based on traction-frame in which the boundary variable is the flux or, equivalently, the derivative of the Helmholtz variable normal to the element boundary.
These include the works presented in reference [24] and the discontinuous Galerkin method evolved from the discontinuous enrichment method [22,23,25-27]. The term "tractionframe" inherits the fact that the boundary variable for elasticity problems is the boundary traction $[13,14,16,24]$. It is also possible to use the displacement-frame in which the boundary and domain variables are both Helmholtz variables in a way analogous to the hybrid-displacement finite element models commonly used to deal with stress singularity problems, see (1), (2) and references [2-4]. Comparing with the traction-frame and other Trefftz methods, a major advantage of using a displacement-frame is that the relevant elements possess the same global dofs as the continuous Galerkin finite element models. Hence, the former elements can be plugged into the standard finite element program framework and the boundary conditions can be specified in exactly the same ways as the continuous Galerkin elements [30,31]. In the frameless Trefftz methods, there exists no boundary or frame variable. Continuity of the domain variable across the interelement boundary and the boundary conditions are directly constrained. Thus, the dofs for the domain variable in all elements enter the global equation. Examples of the frameless methods include the least square method [28,29], wave-based method (WBM) [32-34], etc.

Unlike most of the hybrid-Trefftz finite element models which possess a large number domain modes, the elements to be proposed are based on the displacement-frame and possess only small number of domain modes. They are six-node triangular elements and are equipped with six or seven domain modes per element. Designing "small" hybrid-Trefftz elements, which possess a small number of domain modes, is indeed more challenging than designing "large" elements. In large elements, the boundary modes are plenty and the elements are less sensitive to the choice and the number of domain modes. Accuracy of the "small" elements can vary considerably with respect to the domain modes and their number. Poorly chosen domain modes can also lead to rank deficiency, coordinate variance and connectivity variance of the element matrix $[35,36]$. In the proposed models, the hybrid variational functional analogous to (2) is used. Both the plane-wave solution and the Bessels solution (in the form of $\left.J_{m}(k r) \exp [i m \theta]\right)$ s are employed to construct the domain modes. For full rankness, a minimal of six domain modes are required in the six-node element [37]. For the plane-wave modes, several sets of locally defined directions are attempted and it is found that the ones contain three equispaced directions yield the highest accuracy. For the Bessel solution modes, it will be seen that the first five and seven modes form two invariant bases. However, our experience in formulating quadrilateral elements indicates that the element accuracy is often, if not always, the highest if the number of domains is minimal [31]. In this light, the equispaced directions devised for the plane-wave modes are employed to 
define the sixth Bessel solution mode in addition to the first five modes. Numerical studies indicate that the hybrid-Trefftz elements equipped with six domain modes are typically $50 \%$ less erroneous than the conventional single-field element.

\section{Continuous Galerkin formulation}

Helmholtz equation is often introduced by using the steadystate acoustics. The Helmholtz variable $u$ can be the spatial amplitude of the pressure or the velocity potential. This paper will restrict itself to bounded domain problems. With the problem domain $\Omega$ partitioned into finite elements $\Omega^{e} \mathrm{~s}$, the problem can be summarized as:

(a) Helmholtz equation: $\nabla^{2} u+k^{2} u=0$ in all $\Omega^{e}$

(b) the natural boundary condition: $\mathbf{n}^{T} \nabla u=\bar{t}$ on all $\Gamma_{t}^{e}$

(c) the essential boundary condition: $u=\bar{u}$ and $\delta u=0$ on all $\Gamma_{u}^{e}$

(d) the reciprocity condition: $\left(\mathbf{n}^{T} \nabla u\right)^{+}+\left(\mathbf{n}^{T} \nabla u\right)^{-}=0$ on all $\Gamma_{m}^{e}$

(e) the compatibility condition: $u^{+}=u^{-}$and $\delta u^{+}=\delta u^{-}$ on all $\Gamma_{m}^{e}$

In the above expressions, $\nabla$ is the gradient operator $(\partial / \partial x, \partial / \partial y)^{T}, \nabla^{2}=\nabla^{T} \nabla$ is the Laplace operator, $\mathbf{n}$ is the outward unit normal vector to the element boundary, $k$ is the wave number, $\delta$ is the variational symbol and $\Gamma_{m}^{e}$ is the inter-element boundary. Moreover, ()$^{+}$and ()$^{-}$denote the braced quantities at the two sides of $\Gamma_{m}^{e}$. In the absence of dissipation, $k$ is real. Otherwise, it is complex. For simplicity, it will be assumed as usual that element boundary $\partial \Omega^{e}$ can be partitioned into the non-overlapping portions $\Gamma_{t}^{e}, \Gamma_{u}^{e}$ and $\Gamma_{m}^{e}$, i.e.

$$
\begin{aligned}
& \Gamma_{t}^{e} \cup \Gamma_{u}^{e} \cup \Gamma_{m}^{e}=\partial \Omega^{e} \text { and } \\
& \Gamma_{t}^{e} \cap \Gamma_{u}^{e}=\Gamma_{u}^{e} \cap \Gamma_{m}^{e}=\Gamma_{m}^{e} \cap \Gamma_{t}^{e}=\varnothing .
\end{aligned}
$$

where $\varnothing$ denotes the null set. The elemental variational functional for the continuous Galerkin finite element formulation is well-known to be:

$$
\Pi^{e}=\frac{1}{2} \int_{\Omega^{e}}\left((\nabla u)^{T} \nabla u-k^{2} u^{2}\right) d \Omega-\int_{\Gamma_{t}^{e}} \bar{t} u d \Gamma
$$

in which $u$ is obtained from nodal interpolation. The functional for the problem domain is equal to the sum of all $\Pi^{e}$ s. By invoking (c), (3) and the divergence theorem:

$$
\int_{\Omega^{e}}\left((\nabla f)^{T} \nabla h+h \nabla^{2} f\right) d \Omega=\int_{\partial \Omega^{e}} h\left(\mathbf{n}^{T} \nabla f\right) d \Gamma
$$

for any smooth functions $f$ and $h$ in $\Omega^{e}$, variation of (4) can be written as:

$$
\begin{aligned}
\delta \Pi^{e}= & -\int_{\Omega^{e}}\left(\nabla^{2} u+k^{2} u\right) \delta u d \Omega \\
& +\int_{\Gamma_{t}^{e}}\left(\mathbf{n}^{T} \nabla u-\bar{t}\right) \delta u d \Gamma+\int_{\Gamma_{m}^{e}}\left(\mathbf{n}^{T} \nabla u\right) \delta u d \Gamma
\end{aligned}
$$

The first and second integrals enforce (a) and (b). The last integral when considered jointly with those of the neighborhood elements enforces (d). Conditions (c) and (e) can be easily satisfied by the nodal interpolated $u$ and are taken as prerequisites. As the nodal interpolation is $\mathrm{C}^{0}$ continuous across the element boundary whilst the trial function $u$ and weight $\delta u$ are of the same basis, the formulation is sometimes termed as the continuous Galerkin formulation.

\section{Continuous Galerkin six-node triangle}

Figure 1a shows the six-node triangular element in the global $(x, y)$ coordinate plane. The global coordinates and the field variable $u$ can be yielded by the standard interpolation, i.e.

$$
\begin{aligned}
\left\{\begin{array}{l}
x \\
y
\end{array}\right\} & =\sum_{i=1}^{6} N_{i}\left\{\begin{array}{l}
x_{i} \\
y_{i}
\end{array}\right\} \text { and } \\
u & =\sum_{i=1}^{6} N_{i} u_{i}=\left[N_{1}, \ldots, N_{6}\right]\left\{\begin{array}{l}
u_{1} \\
\vdots \\
u_{6}
\end{array}\right\}=\mathbf{N q}
\end{aligned}
$$

where $N_{1}=(1-s-t)(1-2 s-2 t), N_{2}=s(2 s-1), N_{3}=$ $t(2 t-1), N_{4}=4 s t, N_{5}=4 t(1-s-t), N_{6}=4 s t$ and $(s, t)$ are the area coordinates which vary between 0 and 1 . Nodes 1 to 3 are the corner nodes whereas as nodes 4 to 6 are side nodes. The interpolation matrix $\mathbf{N}$ and the vector of nodal dofs $\mathbf{q}$ are self-defined. With (7) substituted into (4),

$$
\Pi^{e}=\frac{1}{2} \mathbf{q}^{T} \mathbf{K}_{c} \mathbf{q}-\mathbf{f}^{T} \mathbf{q}
$$

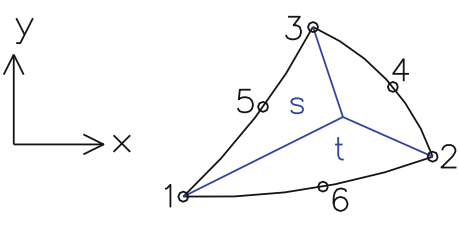

(a)

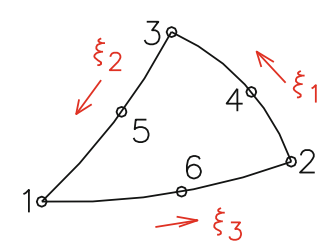

(b)
Fig. 1 The six-node triangular element. a $s$ and $t \in[0,1]$ are the area coordinates and $\mathbf{b} \xi_{1}, \xi_{2}$ and $\xi_{3} \in[-1,+1]$ are the parametric coordinates along the element edges 
in which

$$
\begin{aligned}
\mathbf{K}_{c} & =\int_{\Omega^{e}}\left((\nabla \mathbf{N})^{T}(\nabla \mathbf{N})-k^{2} \mathbf{N}^{T} \mathbf{N}\right) d \Omega \\
& =\int_{0}^{1} \int_{0}^{1-t}\left((\nabla \mathbf{N})^{T}(\nabla \mathbf{N})-k^{2} \mathbf{N}^{T} \mathbf{N}\right) J d s d t \text { and } \\
\mathbf{f} & =\int_{\Gamma_{t}^{e}} \mathbf{N}^{T} \bar{t} d \Gamma .
\end{aligned}
$$

In the element matrix $\mathbf{K}_{c}, J=(\partial x / \partial s)(\partial y / \partial t)-(\partial y / \partial s)$ $(\partial x / \partial t)$ is the Jacobian determinant.

As $\mathbf{N}$ is quadratic in $(s, t)$, the six-point integration rule which is exact up to the fourth order is required to evaluate the element. Though the three-point integration rule which is exact up to the second order also yields rank-sufficient element, it will be seen in the numerical examples that the resultant element accuracy is lower than that of the element evaluated by the six-point integration rule. When the element is subparametric, i.e. its side nodes are mid-side nodes, the interpolated $(x, y)$ are linear in $(s, t)$ and $J$ is a constant.

\section{Hybrid-Trefftz formulation}

In analogous to functional for hybrid-displacement formulation in (1), the following elemental hybrid functional [30,31] can be formed by introducing a second Helmholtz approximation $u_{d}$ to (4):

$$
\begin{aligned}
\Pi_{h}^{e}= & \frac{1}{2} \int_{\Omega^{e}}\left(\left(\nabla u_{d}\right)^{T} \nabla u_{d}-k^{2} u_{d}^{2}\right) d \Omega \\
& +\int_{\partial \Omega^{e}}\left(\mathbf{n}^{T} \nabla u_{d}\right)\left(u_{b}-u_{d}\right) d \Gamma-\int_{\Gamma_{t}^{e}} \bar{t} u_{b} d \Gamma .
\end{aligned}
$$

In the functional, $u_{b}$ and $u_{d}$ are the boundary and domain Helmholtz variables, respectively. Obviously, (9) degenerates into (4) when $u_{b}$ equals $u_{d}$ and $u_{b}$ is denoted by $u$. Again, the functional for the problem domain equals to the sum of all $\Pi_{h}^{e}$ s. Variation of $\Pi_{h}^{e}$ is:

$$
\begin{aligned}
\delta \Pi_{h}^{e}= & \int_{\Omega^{e}}\left(\left(\nabla u_{d}\right)^{T} \nabla \delta u_{d}-k^{2} u_{d} \delta u_{d}\right) d \Omega \\
& +\int_{\partial \Omega^{e}}\left(\left(\mathbf{n}^{T} \nabla \delta u_{d}\right)\left(u_{b}-u_{d}\right)+\left(\mathbf{n}^{T} \nabla u_{d}\right)\left(\delta u_{b}-\delta u_{d}\right)\right) d \Gamma \\
& -\int_{\Gamma_{t}^{e}} \bar{t} \delta u_{b} d \Gamma
\end{aligned}
$$

By invoking the divergence theorem in (5) and assuming that $u_{b}$ satisfies (c) and (e) as prerequisites,

$$
\begin{aligned}
\delta \Pi_{h}^{e}= & -\int_{\Omega^{e}}\left(\nabla^{2} u_{d}+k^{2} u_{d}\right) \delta u_{d} d \Omega \\
& +\int_{\partial \Omega^{e}}\left(\mathbf{n}^{T} \nabla \delta u_{d}\right)\left(u_{b}-u_{d}\right) d \Gamma \\
& +\int_{\Gamma_{t}^{e}}\left(\mathbf{n}^{T} \nabla u_{d}-\bar{t}\right) \delta u_{b} d \Gamma+\int_{\Gamma_{m}^{e}}\left(\mathbf{n}^{T} \nabla u_{d}\right) \delta u_{b} d \Gamma .
\end{aligned}
$$

It can be seen that the second integral enforces the equality of $u_{b}$ and $u_{d}$ along the element boundary $\partial \Omega^{e}$. The first, third and fourth integrals enforce respectively Conditions (a), (b) and (d) in Sect. 2 on $u_{d}$. If $u_{d}$ satisfies the Helmholtz equation, i.e.

$\nabla^{2} u_{d}+k^{2} u_{d}=0$

pointwisely, (9) can be expressed in analogous to (2) as:

$$
\begin{aligned}
\Pi_{h}^{e}= & \int_{\partial \Omega^{e}}\left(-\frac{1}{2}\left(\mathbf{n}^{T} \nabla u_{d}\right) u_{d}+\left(\mathbf{n}^{T} \nabla u_{d}\right) u_{b}\right) d \Gamma \\
& -\int_{\Gamma_{t}^{e}} \bar{t} u_{b} d \Gamma
\end{aligned}
$$

which involves only boundary integrals and the polynomialbased nodal interpolation for $u_{b}$ in (7) can also be used for $u_{b}$, i.e.

$u_{b}=\mathbf{N q}$.

The domain variable extracting from a Trefftz solution set, which satisfies (12), can be expressed as:

$u_{d}=\mathbf{P} \boldsymbol{\beta}$

where $\mathbf{P}$ is the shape function matrix and $\boldsymbol{\beta}$ is the vector of coefficients. The selection of the domain modes will be discussed in later sections. With (14) and (15) substituted into (13),

$\Pi_{h}^{e}=-\frac{1}{2} \boldsymbol{\beta}^{T} \mathbf{H} \boldsymbol{\beta}+\boldsymbol{\beta}^{T} \mathbf{G} \mathbf{q}-\mathbf{f}^{T} \mathbf{q}$.

in which

$\mathbf{H}=\int_{\partial \Omega^{e}}\left(\mathbf{n}^{T} \nabla \mathbf{P}\right)^{T} \mathbf{P} d \Gamma, \quad \mathbf{G}=\int_{\partial \Omega^{e}}\left(\mathbf{n}^{T} \nabla \mathbf{P}\right)^{T} \mathbf{N} d \Gamma$

and $\mathbf{f}$ has been defined in (8). Furthermore, the H-matrix should be symmetric but it may lose its symmetry if it is numerically evaluated. In our programming implementation, $\mathbf{H}$ is symmetrized by averaging itself and its transpose. If the plane wave functions are employed in $u_{d}$ and the element is subparametric, $\mathbf{H}$ and $\mathbf{G}$ can be analytically evaluated 
$[19,21,38,39]$. Variation of (16) with respect to $\boldsymbol{\beta}$ yields:

$$
\mathbf{H} \boldsymbol{\beta}=\mathbf{G q} \text { or } \boldsymbol{\beta}=\mathbf{H}^{-1} \mathbf{G q}
$$

with which the domain variable can be retrieved via (15) as:

$u_{d}=\mathbf{P} \boldsymbol{\beta}=\mathbf{P H}^{-1} \mathbf{G q}$

and $\boldsymbol{\beta}$ can be condensed from $\Pi_{h}^{e}$. The latter becomes:

$\Pi_{h}^{e}=\frac{1}{2} \mathbf{q}^{T}\left(\mathbf{G}^{T} \mathbf{H}^{-1} \mathbf{G}\right) \mathbf{q}-\mathbf{f}^{T} \mathbf{q}=\frac{1}{2} \mathbf{q}^{T} \mathbf{K}_{h} \mathbf{q}-\mathbf{f}^{T} \mathbf{q}$

where element matrix $\mathbf{K}_{h}$ is self-defined. Same as the element matrix $\mathbf{K}_{c}$ arising from the continuous Galerkin formulation, $\mathbf{K}_{h}$ can readily be incorporated into the standard finite element program framework discussed in elementary textbook.

\section{Six-node hybrid-Trefftz elements}

To conduct the boundary integration for the six-node element, three natural coordinates $\xi_{i} \in[-1,+1]$ can be established along the element edges as shown in Fig. $1 \mathrm{~b}$ from the area coordinates $(s, t)$ in (7) by appropriate transformation. Along the $i$-th edge, the boundary variable and the Cartesian coordinates can be interpolated as:

$u_{b}=M_{I} u_{I}+M_{I I} u_{I I}+M_{I I I} u_{I I I}=\mathbf{M}_{i} \mathbf{q}$,

$x=M_{I} x_{I}+M_{I I} x_{I I}+M_{I I I} x_{I I I}$,

$y=M_{I} y_{I}+M_{I I} y_{I I}+M_{I I I} y_{I I I}$

where $M_{I}=\xi_{i}\left(\xi_{i}-1\right) / 2, M_{I I}=1-\xi_{i} \xi_{i}$ and $M_{I I I}=$ $\xi_{i}\left(\xi_{i}+1\right) / 2$ are the interpolation functions of the starting node $\left(\xi_{i}=-1\right)$, middle node $\left(\xi_{i}=0\right)$ and end node $\left(\xi_{i}=\right.$ $+1)$ of the edge, respectively. The interpolation matrix $\mathbf{M}_{i}$ is self-defined and contains only three non-zero entries. In terms of $\xi_{i}$, the differential boundary length and the outward unit normal vector can be expressed as:

$$
\begin{aligned}
& \Gamma=d \xi_{i} \sqrt{\left(\partial x / \partial \xi_{i}\right)^{2}+\left(\partial y / \partial \xi_{i}\right)^{2}}, \\
& \mathbf{n}=\frac{1}{\sqrt{\left(\partial x / \partial \xi_{i}\right)^{2}+\left(\partial y / \partial \xi_{i}\right)^{2}}}\left\{\begin{array}{l}
\partial y / \partial \xi_{i} \\
-\partial x / \partial \xi_{i}
\end{array}\right\}
\end{aligned}
$$

Hence, the H-matrix and G-matrix can be evaluated as:

$$
\begin{aligned}
& \mathbf{H}=\sum_{i=1}^{3} \int_{-1}^{+1}\left(\frac{\partial y}{\partial \xi_{i}} \frac{\partial \mathbf{P}}{\partial x}-\frac{\partial x}{\partial \xi_{i}} \frac{\partial \mathbf{P}}{\partial y}\right)^{T} \mathbf{P} d \xi_{i} \text { and } \\
& \mathbf{G}=\sum_{i=1}^{3} \int_{-1}^{+1}\left(\frac{\partial y}{\partial \xi_{i}} \frac{\partial \mathbf{P}}{\partial x}-\frac{\partial x}{\partial \xi_{i}} \frac{\partial \mathbf{P}}{\partial y}\right)^{T} \mathbf{M}_{i} d \xi_{i} .
\end{aligned}
$$

In designing hybrid elements for stress/structural analyses $[1-5,10,30,31]$, the two common guidelines on the selection of the stress (domain) modes are that the element should be rank sufficient and invariant. Rank sufficiency is guided by (a)

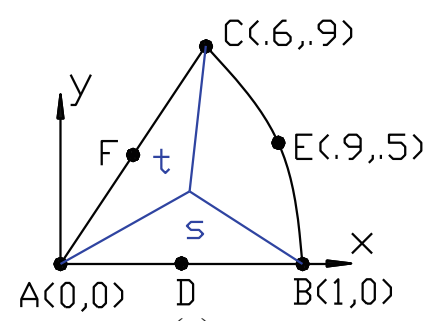

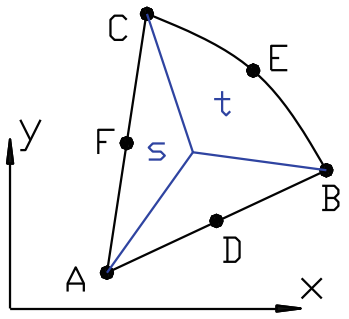

(b)
Fig. 2 The single-element problem for examining the condition number and invariance of the element matrices, $\mathbf{b}$ is obtained from $\mathbf{a}$ by rigid body translation and rotation. Moreover, $A$ is the first element node in a whereas $C$ is the first element node in $\mathbf{b}$

the LBB condition [37]. In the present formulation, it requires the number of domain modes to be greater than or equal to the number of boundary modes. For the present six-node triangular elements problem, the minimal number is six. An element is invariant if the element prediction remains intact when (i) the element is translated, (ii) the element is rotated and (iii) the element connectivity, which defines the directions of the parametric coordinates of the element, is changed [30,31]. For instance, the elements in Fig. 2a, b are identical in geometry and the latter is obtained from the former through translation, rotation and re-declaring the first element node from $\mathrm{A}$ to $\mathrm{C}$. The two elements are expected to possess the same set of eigenvalues. However, if the element model is variant, their eigenvalues would not be identical. Indeed, invariance can be subdivided into (i) translational invariance, (ii) rotational invariance and (iii) connectivity invariance, respectively.

\section{Domain modes from plane-wave solution set}

This and the next sections discuss the selection of the domain modes from the plane-wave and Bessel solution sets, respectively. The plane-wave solution set can be expressed as:

$$
\begin{aligned}
& \left\{p\left(\varphi_{l}\right), l=1,2,3, \ldots\right\} \\
& \quad=\left\{\exp \left[i k r \cos \left(\theta-\varphi_{l}\right)\right], l=1,2,3, \ldots\right\} \\
& \quad=\left\{\exp \left[i k\left(x \cos \varphi_{l}+y \sin \varphi_{l}\right)\right], l=1,2,3, \ldots\right\}
\end{aligned}
$$

where $p$ 's are self-defined, $x=r \cos \theta, y=r \sin \theta$ and $\varphi_{l}$ denotes the propagation direction of the plane-wave. Restricted ourselves to real $k$,

$$
\begin{aligned}
& \operatorname{Re}\left(p\left(\varphi_{l} \pm \pi\right)\right)=\cos \left(k x \cos \varphi_{l}+k y \sin \varphi_{l}\right)=\operatorname{Re}\left(p\left(\varphi_{l}\right)\right), \\
& \operatorname{Im}\left(p\left(\varphi_{l} \pm \pi\right)\right)=-\sin \left(k x \cos \varphi_{l}+k y \sin \varphi_{l}\right)=-\operatorname{Im}\left(p\left(\varphi_{l}\right)\right) .
\end{aligned}
$$

Hence, once a direction is included, the reverse direction should not be employed so as to avoid linear dependence. Otherwise, both the $\mathrm{H}$ - and G-matrices will be rank deficient. 
It should be remarked that the linear dependence will not be valid for non-zero $\operatorname{Im}(k)$.

When another set of coordinates $(\widehat{x}, \widehat{y})=\left(x-x_{c}, y-y_{c}\right)$, where $\left(x_{c}, y_{c}\right)$ represents a translational shift of the coordinate system, is employed in replacement of $(x, y)$, a component in (23) becomes

$$
\begin{aligned}
\widehat{p}\left(\varphi_{l}\right)= & \exp \left[i k\left(\widehat{x} \cos \varphi_{l}+\widehat{y} \sin \varphi_{l}\right)\right] \\
= & \exp \left[-i k\left(x_{c} \cos \varphi_{l}+y_{c} \sin \varphi_{l}\right)\right] \\
& \times \exp \left[i k\left(x \cos \varphi_{l}+y \sin \varphi_{l}\right)\right] \\
= & \exp \left[-i k\left(x_{c} \cos \varphi_{l}+y_{c} \sin \varphi_{l}\right)\right] \widehat{p}\left(\varphi_{l}\right)
\end{aligned}
$$

which possesses the same basis as $p\left(\varphi_{l}\right)$. Hence, the planewave modes are translational invariant. Nevertheless, if the element size is very small compared with its distance from the coordinate origin, numerical error may arise. Therefore, it is a common practice to use a point $\left(x_{c}, y_{c}\right)$ local to the element to define $(\widehat{x}, \widehat{y})$ which are in turn used in defining the domain modes [1-5, 10,22,23,25,27].

\subsection{Directions for elements TP1, TP2 and TP3}

To secure the rank sufficiency, three directions or six plane modes are needed. A few simple sets of three local directions can be defined, they include:

- the three directions perpendicular to the three straight lines joining the corner nodes of the element, see Fig. 3a, the resultant element will be denoted as TP1;

- the three directions along straight lines joining the corner nodes and side nodes of the element, see Fig. 3b, the resultant element will be denoted as TP2;

- the three directions along the straight lines joining the corner nodes of the element, see Fig. 3c, the resultant element will be denoted as TP3.
The first and third sets have also been considered by Grosu and Harari [23] in their triangular elements with discontinuous enrichment. For elements TP1, TP2 and TP3, the following local point $\left(x_{c}, y_{c}\right)$ inside the element is employed to define the local coordinates $(\hat{x}, \widehat{y})$ :

$x_{c}=\left(x_{1}+x_{2}+x_{3}\right) / 3$ and $y_{c}=\left(y_{1}+y_{2}+y_{3}\right) / 3$

in the domain modes. It should be remarked that the choice of the local point $\left(x_{c}, y_{c}\right)$ does not affect the basis of the domain modes, see (25).

\subsection{Local equispaced directions}

In previous computational models that employ the planewave solution, $\varphi_{l} \mathrm{~s}$ are often picked at equal interval [17-21,24,29]. However, if one simply picks three fixed equispaced directions such as $\varphi_{1}=0, \varphi_{2}=2 \pi / 3$ and $4 \pi / 3$, the element would be rotational variant. In this light, the point $\mathrm{C}\left(x_{c}, y_{c}\right)$ is sought such that $\mathrm{C} 1, \mathrm{C} 2$ and $\mathrm{C} 3$ are equispaced as shown in Fig. 3d and the propagation directions would be taken as:

$\varphi_{l}=\arctan \left(y_{l}-y_{c}, x_{l}-x_{c}\right)$ for $l=1,2$ and 3.

The condition can be mathematically expressed as:

$$
\frac{|\overrightarrow{C 1} \times \overrightarrow{C 2}|}{|\overrightarrow{C 1}| \cdot|\overrightarrow{C 2}|}=\tan \frac{2 \pi}{3}, \frac{|\overrightarrow{C 2} \times \overrightarrow{C 3}|}{|\overrightarrow{C 2}| \cdot|\overrightarrow{C 3}|}=\tan \frac{2 \pi}{3}
$$

or

$$
\begin{aligned}
& \frac{\left(y_{1}-y_{c}\right)\left(x_{2}-x_{c}\right)-\left(x_{1}-x_{c}\right)\left(y_{2}-y_{c}\right)}{\left(x_{1}-x_{c}\right)\left(x_{2}-x_{c}\right)+\left(y_{1}-y_{c}\right)\left(y_{2}-y_{c}\right)}=\sqrt{3}, \\
& \frac{\left(y_{2}-y_{c}\right)\left(x_{3}-x_{c}\right)-\left(x_{2}-x_{c}\right)\left(y_{3}-y_{c}\right)}{\left(x_{2}-x_{c}\right)\left(x_{3}-x_{c}\right)+\left(y_{2}-y_{c}\right)\left(y_{3}-y_{c}\right)}=\sqrt{3}
\end{aligned}
$$

Fig. 3 Directions of the plane-wave modes employed in a TP1, b TP2, c TP3, d TP and e TP*. The local coordinate origins and directions in $\mathbf{d}$ and $\mathbf{e}$ are also employed in the cylindrical-wave modes of TJ6 and $\mathrm{TJ}^{*}$

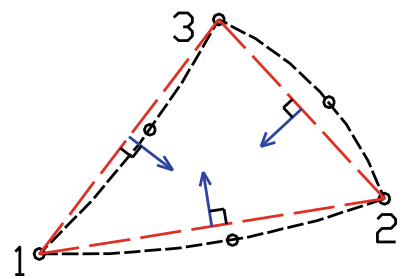

(a)

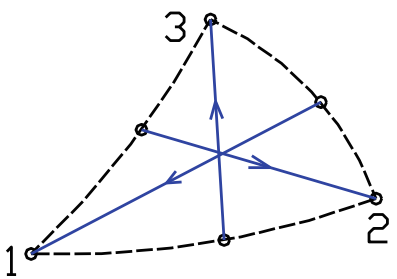

(b)

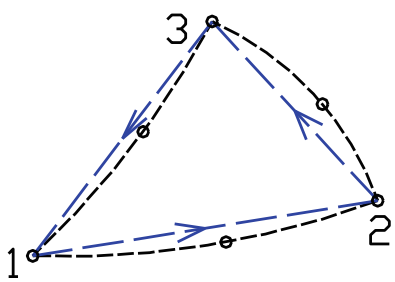

(c)

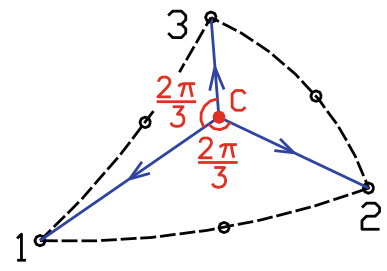

(d)

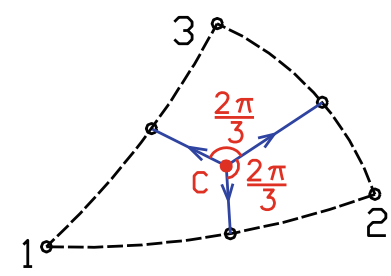

(e) 
By solving them,

$$
\begin{aligned}
x_{c}= & \frac{1}{D} \sum_{i=1}^{3}\left(\frac{\left(x_{i}^{2}-3 y_{i}^{2}\right)\left(y_{j}-y_{k}\right)-4 x_{i} y_{i}\left(x_{j}-x_{k}\right)}{2 \sqrt{3}}\right. \\
& \left.+\frac{\left(x_{i}^{2}+y_{i}^{2}\right)\left(x_{j}+x_{k}\right)}{2}-x_{i} y_{j} y_{k}\right)-\frac{3 x_{1} x_{2} x_{3}}{D}, \\
y_{c}= & \frac{1}{D} \sum_{i=1}^{3}\left(\frac{4 x_{i} y_{i}\left(y_{j}-y_{k}\right)-\left(y_{i}^{2}-3 x_{i}^{2}\right)\left(x_{j}-x_{k}\right)}{2 \sqrt{3}}\right. \\
& \left.+\frac{\left(x_{i}^{2}+y_{i}^{2}\right)\left(y_{j}+y_{k}\right)}{2}-y_{i} x_{j} x_{k}\right)-\frac{3 y_{1} y_{2} y_{3}}{D}
\end{aligned}
$$

where

$$
\begin{aligned}
& D=\sum_{i=1}^{3}\left(\frac{\left(x_{i}-x_{j}\right)^{2}+\left(y_{i}-y_{j}\right)^{2}}{2}+\sqrt{3} x_{i}\left(y_{j}-y_{k}\right)\right), \\
& j=\bmod (i, 3)+1 \text { and } k=\bmod (i+1,3)+1 .
\end{aligned}
$$

The resultant element will be denoted as TP.

By changing subscripts $(1,2,3)$ to subscripts $(4,5,6)$, (29) gives another local coordinate origin $\mathrm{C}\left(x_{c}, y_{c}\right)$ such that C4, C5 and C6 constitute any set of equispaced directions as shown in Fig. 3e. In other words,

$\varphi_{l}=\arctan \left(y_{l+3}-y_{c}, x_{l+3}-x_{c}\right)$ for $l=1,2$ and 3.(30)

The resultant element will be denoted as TP*. It would be seen in the numerical examples, that TP and TP* are often more accurate than TP1, TP2 and TP3.

\section{Domain modes from Bessel solution set}

This section considers the domain modes selected from the Bessel solution set. As specified earlier, the elements to be proposed are for bounded domain problems. Provided that one is expanding the solution about an interior point $\left(x_{c}, y_{c}\right)$ of the element and there is no source or singular points within the element domain or on the element boundary, the solution set should be restricted to Bessel functions of the first kind and the singular solution set based on the second kind can be excluded $[24,40]$. For the same reasons, one adopts the plane wave solution set which should not be used to approximate singular solutions. The non-singular Bessel solution set is:

$$
\left\{J_{m}(k \widehat{r}) \exp (i m \hat{\theta}), \quad m=0,1,2, \ldots\right\}
$$

where $J_{m}$ is the $m$-th order Bessel function of the first kind,

$$
\begin{aligned}
& \widehat{r}=\sqrt{\hat{x}^{2}+\hat{y}^{2}}, \quad \hat{\theta}=\arctan (\hat{y}, \widehat{x}), \\
& \widehat{x}=x-x_{c}, \quad \hat{y}=y-y_{c}
\end{aligned}
$$

and $\arctan ($,$) returns the principal angle of its arguments.$ Both the real and imaginary parts of the members in the solution set can be taken to be domain modes. To secure the translational and connectivity invariance, $\left(x_{c}, y_{c}\right)$ should be symmetric with respect to the corner nodes and/or the side nodes. When the element is rotated by any angle $\alpha$, the real and imaginary modes associated with $J_{m}$ for real $k$ can be expanded as:

$$
\begin{aligned}
& \operatorname{Re}\left(J_{m}(k \widehat{r}) \exp [i m(\widehat{\theta}+\alpha)]\right)=\operatorname{Re}\left[J_{m}(k \widehat{r}) \exp (i m \widehat{\theta})\right] \\
& \quad \times \cos m \alpha-\operatorname{Im}\left[J_{m}(k \widehat{r}) \exp (i m \widehat{\theta})\right] \sin m \alpha, \\
& \operatorname{Im}\left(J_{m}(k \widehat{r}) \exp [i m(\widehat{\theta}+\alpha)]\right)=\operatorname{Im}\left[J_{m}(k \widehat{r}) \exp (i m \widehat{\theta})\right] \\
& \quad \times \cos m \alpha-\operatorname{Re}\left[J_{m}(k \widehat{r}) \exp (i m \widehat{\theta})\right] \sin m \alpha
\end{aligned}
$$

which, therefore, constitute a rotational invariant basis. Hence,

$$
\begin{gathered}
\left\{J_{m}(k \widehat{r}) \exp (i m \hat{\theta})\right\}=\left\{\operatorname{Re}\left(J_{m}(k \widehat{r}) \exp (i m \hat{\theta})\right),\right. \\
\left.\operatorname{Im}\left(J_{m}(k \widehat{r}) \exp (i m \widehat{\theta})\right)\right\} \quad \text { where } m=0,1,2, \ldots
\end{gathered}
$$

is an invariant basis which possesses translational, rotational and connectivity invariance. It should be remarked that the sets in (31) contain infinite number of members arising from all positive integer $m$ whilst the set in (34) contains only the member(s) of a specific $m$.

\subsection{Element with seven Bessel solution modes}

Starting from the zeroth order Bessel mode, the following shape function matrix of the domain modes:

$$
\begin{aligned}
& \mathbf{P}=\left[J_{0}(k \widehat{r}), J_{1}(k \widehat{r}) \cos \widehat{\theta}, J_{1}(k \widehat{r}) \sin \widehat{\theta},\right. \\
& \left.J_{2}(k \widehat{r}) \cos 2 \widehat{\theta}, J_{2}(k \widehat{r}) \sin 2 \widehat{\theta}, J_{3}(k \widehat{r}) \cos 3 \widehat{\theta}, J_{3}(k \widehat{r}) \sin 3 \widehat{\theta}\right]
\end{aligned}
$$

include all the zeroth to third order Bessel modes. Several local coordinates origins $\left(x_{c}, y_{c}\right)$ observe the symmetry discussed in above (32) including the average coordinates of the three corner nodes, the average coordinates of the three side nodes as well as those employed by TP and TP*. The predictions are virtually insensitive to the above choices of the local coordinate origins. For simplicity, the results to be presented are computed by taking $\left(x_{c}, y_{c}\right)$ to be the average coordinates of the three corner nodes. The resulting element will be denoted as TJ7 which is invariant and is also checked to be rank sufficient.

\subsection{Elements with six Bessel solution modes}

The minimal number of domain modes is six for rank sufficient. To attain this number, one of the $J_{3}$ modes in (35) may be dropped. However, the resultant element will no longer be rotational invariant. The rotational invariance can be restored 
when a local direction is employed to define the remaining $J_{3}$-mode. If, for instance, the running direction along a line joining any two nodes is adopted, the resulting element would be connectivity variant. In this light, the local directions are chosen to be the ones in (27) and the coordinate origin $\mathrm{C}\left(x_{c}, y y_{c}\right)$ be that in (29). As the directions differ by $\pm 2 \pi / 3$,

$$
\begin{aligned}
& \cos 3\left(\widehat{\theta}-\phi_{1}\right)=\cos 3\left(\widehat{\theta}-\phi_{2}\right)=\cos 3\left(\widehat{\theta}-\phi_{3}\right) \\
& \text { and } \sin 3\left(\widehat{\theta}-\phi_{1}\right)=\sin 3\left(\widehat{\theta}-\phi_{2}\right)=\sin 3\left(\widehat{\theta}-\phi_{3}\right)
\end{aligned}
$$

Hence, both

$$
\begin{aligned}
& J_{3}(k \widehat{r}) \cos 3(\widehat{\theta}-\phi) \text { and } J_{3}(k \widehat{r}) \sin 3(\widehat{\theta}-\phi) \\
& \text { for } \phi \text { equal to any of } \phi_{1}, \phi_{2} \text { and } \phi_{3}
\end{aligned}
$$

are invariant. It can be noted that the $J_{3}$-cos term can raise the rank of the element formulated with the first five modes in (35) but the $J_{3}$-sin term cannot. Hence, the following shape function matrix of the domain modes is devised:

$$
\begin{aligned}
\mathbf{P}= & {\left[J_{0}(k \widehat{r}), J_{1}(k \widehat{r}) \cos \widehat{\theta}, J_{1}(k \widehat{r}) \sin \widehat{\theta}, J_{2}(k \widehat{r}) \cos 2 \widehat{\theta},\right.} \\
& \left.J_{2}(k \widehat{r}) \sin 2 \widehat{\theta}, J_{3}(k \widehat{r}) \cos 3(\widehat{\theta}-\phi)\right]
\end{aligned}
$$

The resultant element will be denoted as TJ6.

Similarly, one can also employ the local coordinate origin $\mathrm{C}\left(x_{c}, y_{c}\right)$ of TP6* described at the end of Section 4.2 and take $\phi$ to be any of $\phi_{4}, \phi_{5}$ and $\phi_{6}$. Again, the shape function matrix in (38) yields a rank sufficient element whereas it does not if $\cos 3(\widehat{\theta}-\phi)$ is replaced by $\sin 3(\widehat{\theta}-\phi)$.

\section{Numerical examples}

Before proceeding to the numerical examples, the following list summarizes the discussed six-node triangular element models:

- TC - the continuous Galerkin element evaluated by the six-point integration rule, see (8);

- $\mathrm{TC}^{*}$ - the continuous Galerkin element evaluated by the three-point integration rule, see (8);

- TP1-the element formulated with six plane-wave modes whose directions are perpendicular to the straight lines joining the corner nodes, see Section 6.1 and Fig. 3a;

- TP2 - the element formulated with six plane-wave modes whose directions are along the straight lines joining the corner and side nodes, see Section 6.1 and Fig. 3b;

- TP3 - the element formulated with six plane-wave modes whose directions are along the straight lines joining the corner nodes, see Section 6.1 and Fig. 3c;

- TP-the element formulated with six equispaced planewave modes with directions along the straight lines joining a local coordinate origin $\mathrm{C}$ and the corner nodes, see Section 6.2 and Fig. 3d;
- TP*-the element formulated with six equispaced planewave modes with directions along the straight lines joining a local element point and the side nodes, see the last paragraph in Section 6.2 and Fig. 3e;

- TJ7-the element formulated with seven Bessel solution modes, Section 7.1;

- TJ6-the element formulated with six Bessel solution modes and the $J_{3}$ mode is defined by any one of the wave propagation directions of TP, see Section 7.2 and Fig. 3d;

- TJ6*-the element formulated with six Bessel solution modes and the $J_{3}$ mode is defined by any one of the wave propagation directions of TP*, see the last paragraph of Section 7.2 and Fig. 3e.

The above list contains too many element models. To enhance the graphical clarity in portraying the numerical predictions, the following three models would be excluded in the subsequent comparisons:

- $\mathrm{TP}^{*}$-This element is marginally less accurate than $\mathrm{TP}$ and its predictions are graphically indistinguishable from those of TP.

- TJ6*-This element is marginally less accurate than TJ6 and its predictions are graphically indistinguishable from those of TJ6.

- TP3-The problem is often erroneous compared with the other elements. The cause is traced to be the ill-conditioning of the element matrix. Indeed, the element matrix is rank deficient when the element is a subparametric equilateral triangle. With the element side length denoted by $L$, the null space of the element matrix can be derived to be:

$$
\left[\left\{u_{c}, u_{c}, u_{c}, u_{s}, u_{s}, u_{s}\right\}^{T}\right]
$$

in which $u_{c}=-32+8 k L \sin (k L / 2)+32 k L \cos (k L / 2)$ and $u_{s}=u_{c}+(k L)^{2}[1-\cos (k L / 2)]$ are the values at the corner and side nodes, respectively.

In the cases of equilateral subparametric element, it can noted that TP1, TP2, TP and TP* are identical whilst TJ6 and TJ6* are identical.

Unless stated otherwise, the boundary integrations leading to the hybrid-Trefftz element matrices and for implementing the natural boundary of all elements are evaluated by the three-point quadrature. The following normalized and relative errors are defined:

$$
\begin{aligned}
\text { Normalized error } & =\left(\frac{\int_{\Omega}\left(u-u^{\text {exact }}\right)^{2} d \Omega}{\int_{\Omega}\left(u^{\text {exact }}\right)^{2} d \Omega}\right)^{1 / 2} \\
& =\left(\frac{\sum_{e} \int_{\Omega^{e}}\left(u-u^{\text {exact }}\right)^{2} d \Omega}{\sum_{e} \int_{\Omega^{e}}\left(u^{\text {exact }}\right)^{2} d \Omega}\right)^{1 / 2}
\end{aligned}
$$


Table $1|\alpha|_{\max },|\alpha|_{\min }$ and $N_{c}$ of TC under different domain integration rules

\begin{tabular}{|c|c|c|c|c|c|c|c|c|c|}
\hline & \multicolumn{3}{|c|}{ Three-point rule } & \multicolumn{3}{|c|}{ Six-point rule } & \multicolumn{3}{|c|}{ Seven-point rule } \\
\hline & $|\alpha|_{\max }$ & $|\alpha|_{\min }$ & $N_{c}$ & $|\alpha|_{\max }$ & $|\alpha|_{\min }$ & $N_{c}$ & $|\alpha|_{\max }$ & $|\alpha|_{\min }$ & $N_{c}$ \\
\hline$k=1.25$ & 3.205 & 0.147 & 20.81 & 3.150 & 0.147 & 21.37 & 3.150 & 0.147 & 21.38 \\
\hline$k=2.5$ & 3.137 & 0.241 & 13.02 & 2.952 & 0.252 & 11.72 & 2.953 & 0.252 & 11.73 \\
\hline
\end{tabular}

Table $2|\alpha|_{\max },|\alpha|_{\min }$ and $N_{c}$ of the multi-field elements under different orders of 1D quadrature

\begin{tabular}{|c|c|c|c|c|c|c|c|c|c|}
\hline & \multicolumn{3}{|c|}{ Third order quadrature } & \multicolumn{3}{|c|}{ Fourth order quadrature } & \multicolumn{3}{|c|}{ Fifth order quadrature } \\
\hline & $|\alpha|_{\max }$ & $|\alpha|_{\min }$ & $N_{c}$ & $|\alpha|_{\max }$ & $|\alpha|_{\min }$ & $N_{c}$ & $|\alpha|_{\max }$ & $|\alpha|_{\min }$ & $N_{c}$ \\
\hline \multicolumn{10}{|l|}{ TP1 } \\
\hline$k=1.25$ & 3.085 & 0.148 & 20.68 & 3.054 & 0.148 & 20.66 & 3.054 & 0.148 & 20.66 \\
\hline$k=2.5$ & 2.870 & 0.240 & 11.99 & 2.859 & 0.239 & 11.97 & 2.859 & 0.239 & 11.97 \\
\hline \multicolumn{10}{|l|}{$\mathrm{TP} 2$} \\
\hline$k=1.25$ & 3.060 & 0.148 & 20.70 & 3.056 & 0.148 & 20.67 & 3.056 & 0.148 & 20.67 \\
\hline$k=2.5$ & 2.879 & 0.241 & 11.97 & 2.867 & 0.240 & 11.92 & 2.868 & 0.240 & 11.93 \\
\hline \multicolumn{10}{|l|}{ TP3 } \\
\hline$k=1.25$ & 3.082 & 0.013 & 231.4 & 3.084 & 0.012 & 259.5 & 3.084 & 0.012 & 257.4 \\
\hline$k=2.5$ & 2.892 & 0.012 & 236.8 & 2.898 & 0.010 & 281.5 & 2.898 & 0.010 & 279.9 \\
\hline \multicolumn{10}{|l|}{ TP } \\
\hline$k=1.25$ & 3.059 & 0.148 & 20.69 & 3.055 & 0.148 & 20.66 & 3.055 & 0.148 & 20.66 \\
\hline$k=2.5$ & 2.876 & 0.240 & 11.97 & 2.864 & 0.240 & 11.94 & 2.865 & 0.240 & 11.94 \\
\hline \multicolumn{10}{|l|}{$\mathrm{TP}^{*}$} \\
\hline$k=1.25$ & 3.059 & 0.148 & 20.69 & 3.055 & 0.148 & 20.66 & 3.055 & 0.148 & 20.67 \\
\hline$k=2.5$ & 2.876 & 0.240 & 11.97 & 2.864 & 0.240 & 11.94 & 2.865 & 0.240 & 11.94 \\
\hline \multicolumn{10}{|l|}{$\mathrm{TJ} 7$} \\
\hline$k=1.25$ & 3.090 & 0.148 & 20.90 & 3.091 & 0.148 & 20.91 & 3.091 & 0.148 & 20.91 \\
\hline$k=2.5$ & 2.909 & 0.243 & 11.99 & 2.910 & 0.242 & 12.01 & 2.910 & 0.242 & 12.01 \\
\hline \multicolumn{10}{|l|}{ TJ6 } \\
\hline$k=1.25$ & 3.067 & 0.150 & 20.45 & 3.067 & 0.150 & 20.42 & 3.067 & 0.150 & 20.42 \\
\hline$k=2.5$ & 2.877 & 0.243 & 11.85 & 2.876 & 0.242 & 11.87 & 2.876 & 0.242 & 11.87 \\
\hline \multicolumn{10}{|l|}{ TJ6* } \\
\hline$k=1.25$ & 3.062 & 0.148 & 20.71 & 3.059 & 0.148 & 20.69 & 3.059 & 0.148 & 20.69 \\
\hline$k=2.5$ & 2.879 & 0.243 & 11.87 & 2.878 & 0.242 & 11.88 & 2.878 & 0.242 & 11.88 \\
\hline
\end{tabular}

and

$$
\begin{aligned}
\text { Relative error } & =\left(\frac{\int_{\Omega}\left(u-u^{\text {exact }}\right)^{2} d \Omega}{\int_{\Omega}\left(u^{\mathrm{TC}}-u^{\text {exact }}\right)^{2} d \Omega}\right)^{1 / 2} \\
& =\left(\frac{\sum_{e} \int_{\Omega^{e}}\left(u-u^{\text {exact }}\right)^{2} d \Omega}{\sum_{e} \int_{\Omega^{e}}\left(u^{\mathrm{TC}}-u^{\text {exact }}\right)^{2} d \Omega}\right)^{1 / 2}
\end{aligned}
$$

where $u^{\text {exact }}$ denotes the exact solution and $u^{\mathrm{TC}}$ denotes the prediction of the continuous Galerkin TC. In the hybridTrefftz elements, $u_{d}$ is the prediction within the element domain and, thus, $u$ is replaced by $u_{d}$ in the above two error
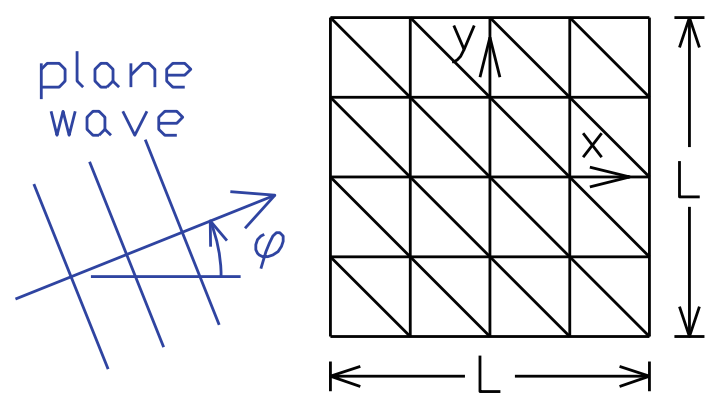

Fig. 4 The plane wave-problem in which the exact solution is $u^{\text {exact }}=$ $\cos (k x \cos \varphi+k y \sin \varphi)$. The figure shows a $L \times L$ problem domain modeled by the $4 \times 4$ mesh which contains $2 \times(4 \times 4)$ triangular elements. All boundaries are prescribed with the natural boundary condition 
measures. In both errors, the domain integrals are evaluated by the six-point integration rule. Furthermore, the number of nodal spacings per wavelength

$N_{n}=$ wave length/nodal spacing $=2 \pi /(k h)$,

will be specified in the examples. In (42), $h$ denotes the nodal spacing. For conventional element models, many literatures recommend $N_{n}>10$ (see, e.g., [19,38] among others) for the continuous Galerkin elements.

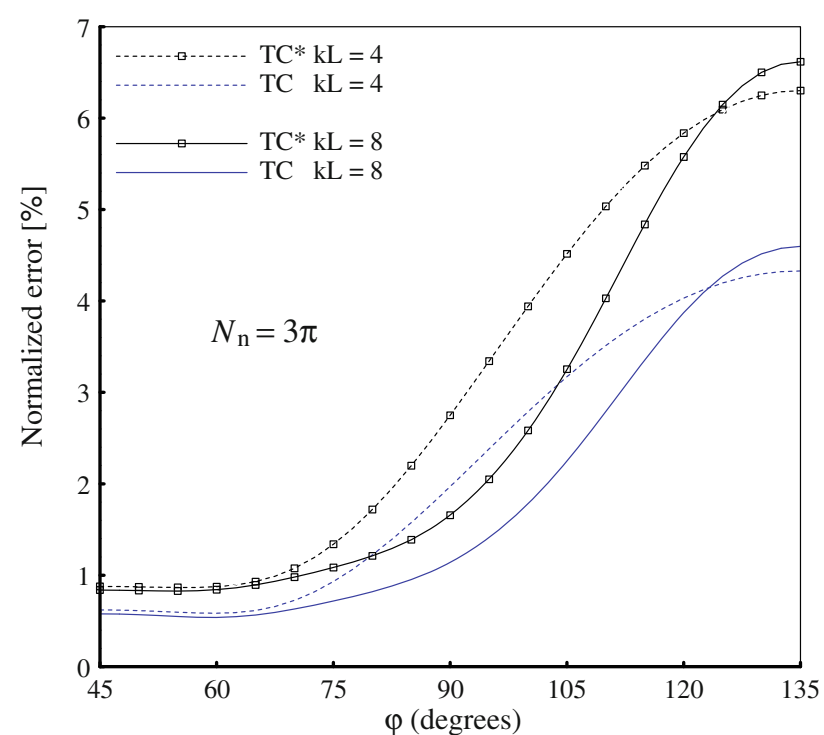

(a)

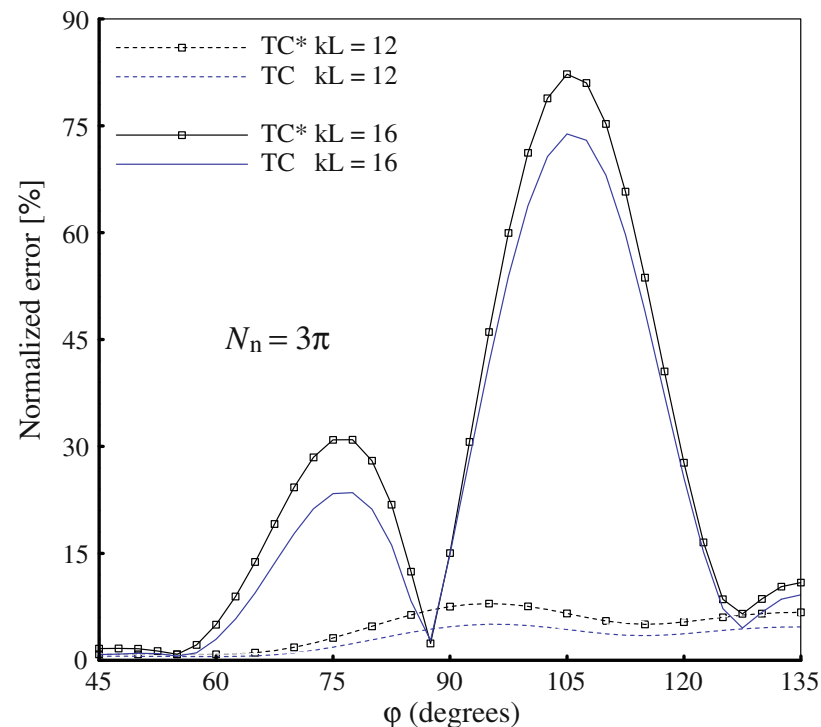

(b)

Fig. 5 Normalized errors of TC* (integrated by the three-point rule) and TC (integrated by the six-point rule) in the plane-wave problem, see Fig. 4. $k L=4,8,12$ and 16 are considered. The number of nodal spacings per wavelength $N_{n}$ is fixed at $3 \pi$.

\subsection{Condition number versus integration order}

The primary purpose of this test is to identify the adequate order of the integration rule for evaluating the element matrices by examining the change of the condition number of the element matrix with respect to the integration order. The element in Fig. 2a is considered. Nodes $\mathrm{D}$ and $\mathrm{F}$ bisect $\mathrm{AB}$ and $\mathrm{AC}$, respectively. The element edge lengths are $1, \sqrt{ } 0.97$ and $\sqrt{ } 1.17$ which gives an average nodal spacing of $\sim 0.51$. Two wavenumbers $k=1.25$ and 2.5 are considered. The relevant $N_{n} \mathrm{~s}$ are approximately equal to 10 and

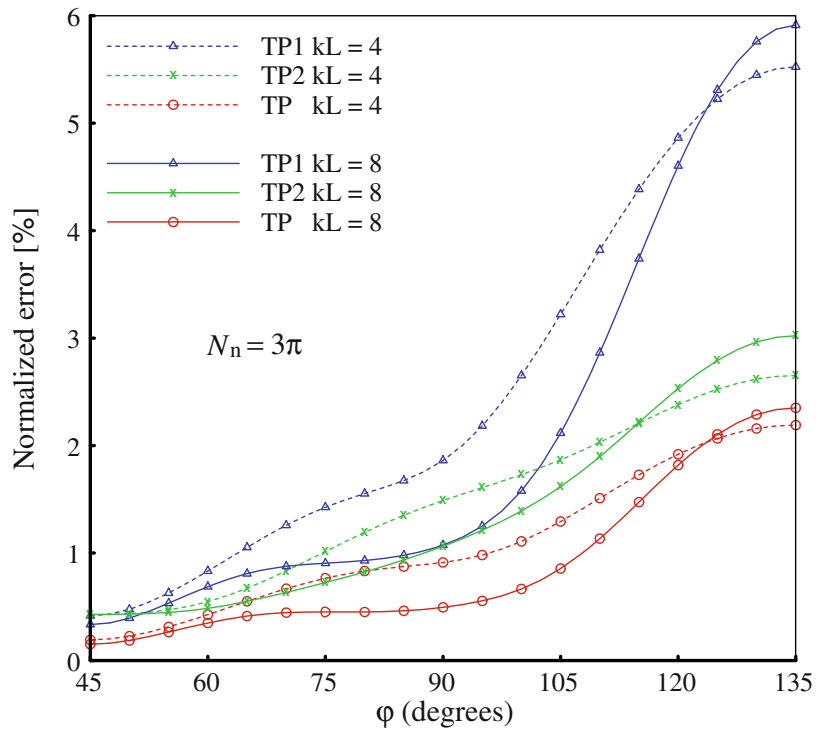

(a)

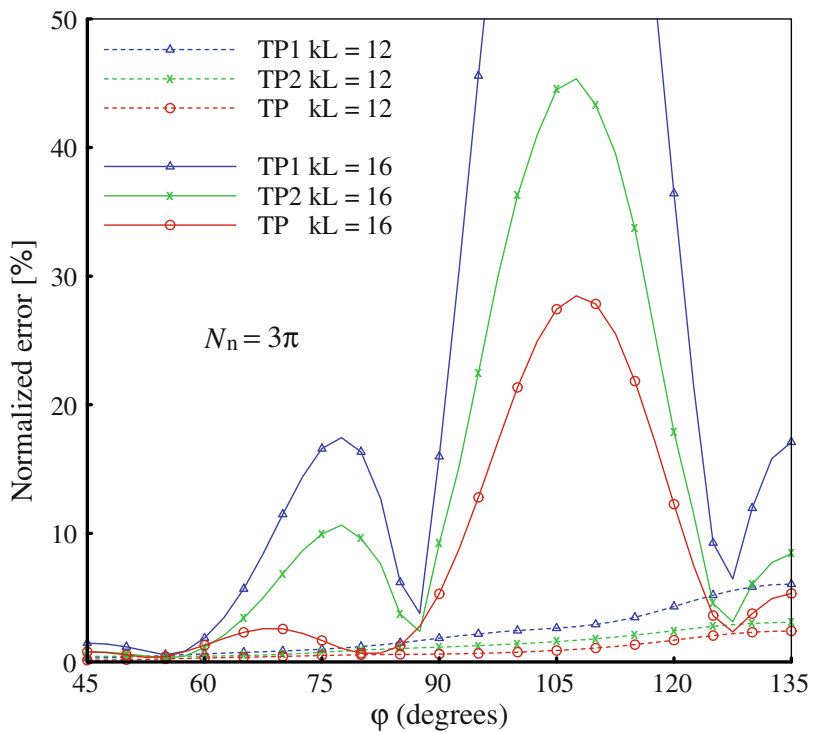

(b)

Fig. 6 Normalized errors of elements based on the plane-wave modes in the plane-wave problem, see Fig. 4. $k L=4,8,12$ and 16 are considered. The number of nodal spacings per wavelength $N_{n}$ is fixed at $3 \pi$. 
5, respectively. Let $|\alpha|$ denote the eigenvalue magnitude of the element matrix, $|\alpha|_{\max },|\alpha|_{\min }$ and the condition number $N_{c}\left(=|\alpha|_{\text {max }} /|\alpha|_{\text {min }}\right)$ are computed. Table 1 lists the computed values of TC when the element matrix is evaluated by three-point, six-point and seven-point domain integration rules [41]. There are two three-point rules and only the more popular one, in which all the sampling points are away from the element boundary, is considered. It can be seen that $N_{c}$ changes considerably when the integration changes from three-point to six-point rule. This point is more obviously when the wavenumber is larger. The observation echoes the previous discussion that the three-point rule can yield a rank sufficient element (otherwise, $|\alpha|_{\text {min }}$ equals zero) whilst the six-point rule is required to integrate the element matrix exactly for subparametric geometry.

Table 2 lists $|\alpha|_{\text {max }},|\alpha|_{\text {min }}$ and $N_{c}$ of TP1, TP2, TP3, TP, TP*, TJ7, TJ6, TJ6* when the boundary integrations leading to the element matrices are evaluated by the third, fourth and fifth order quadratures. When the second order quadrature is employed, $|\alpha|_{\min }$ equals zero which indicate rank deficient. On the other hand, $N_{c}$ s of all elements change by less than $0.5 \%$ when the quadrature order is raised from three to four or five for both values of $k$. The minute changes indicate that the third order of quadrature is sufficient. This deduction is also confirmed in other numerical examples. It has been mentioned that TP3 is rank deficient when the element is an equilateral and subparametric. For the present distorted element geometry, TP3 is rank sufficient but its condition number is ten times larger than those of the other elements.

\subsection{Invariant tests}

The element in Fig. $2 \mathrm{~b}$ is obtained from that in Fig. 2a by translation, rotation and change of connectivity. Other combinations of translation, rotation and connectivity have also been attempted. Under all settings, all the element eigenvalues remain unchanged. The invariance (comprising translational, rotational and connectivity invariance) of all elements are confirmed.

\subsection{Plane wave problem}

Figure 4 depicts a $L \times L$ problem domain modelled by the $4 \times 4$ mesh which contains $2 \times 4 \times 4$ triangular elements and $L$ is set to be 2 . The domain boundary is prescribed with the natural boundary condition with respect to the following plane wave solution:

$u^{\text {exact }}=\cos (k x \cos \varphi+k y \sin \varphi)$

where $\varphi$ is the wave propagation direction. Owing to the mesh topology and domain geometry, the result for $0 \leq \varphi \leq 90^{\circ}$ is symmetric about $\varphi=45^{\circ}$ whereas the result for $90^{\circ} \leq$ $\varphi \leq 180^{\circ}$ is symmetric about $\varphi=135^{\circ}$. For conciseness, only the result for $45^{\circ} \leq \varphi \leq 135^{\circ}$ would be shown.

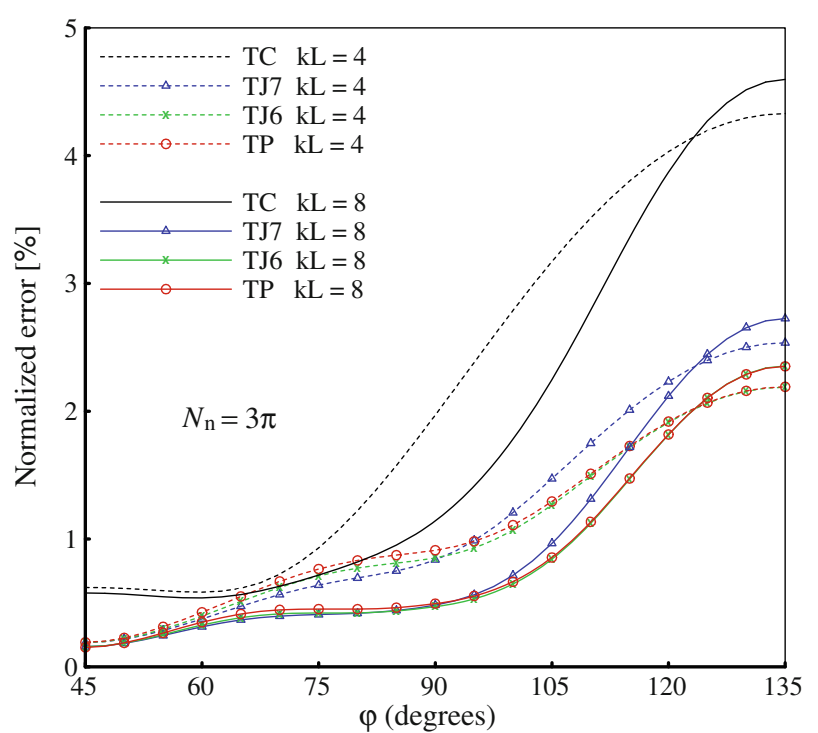

(a)

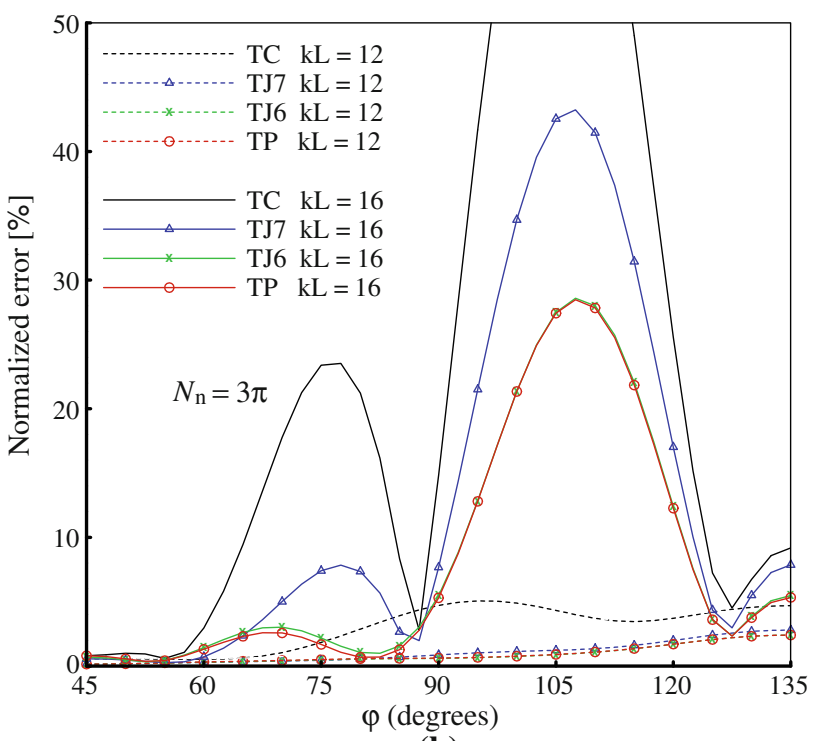

(b)

Fig. 7 Normalized errors of TC, TJ7, TJ6 and TP in the plane-wave problem, see Fig. 4. $k L=4,8,12$ and 16 are considered. The number of nodal spacings per wavelength $N_{n}$ is fixed at $3 \pi$.

Table 3 Average relative errors for results in Figs. 5a to $7 \mathrm{~b}$ on the plane-wave problem

\begin{tabular}{llllllll}
\hline$k L($ Mesh $)$ & TC* & TC & TP1 & TP2 & TP & TJ7 & TJ6 \\
\hline $4(3 \times 3)$ & 1.44 & 1 & 1.21 & 0.79 & 0.55 & 0.54 & 0.52 \\
$8(6 \times 6)$ & 1.48 & 1 & 1.10 & 0.85 & 0.49 & 0.50 & 0.47 \\
$12(9 \times 9)$ & 1.56 & 1 & 0.84 & 0.58 & 0.34 & 0.37 & 0.33 \\
$16(12 \times 12)$ & 1.31 & 1 & 1.84 & 1.03 & 0.41 & 0.51 & 0.42 \\
\hline
\end{tabular}




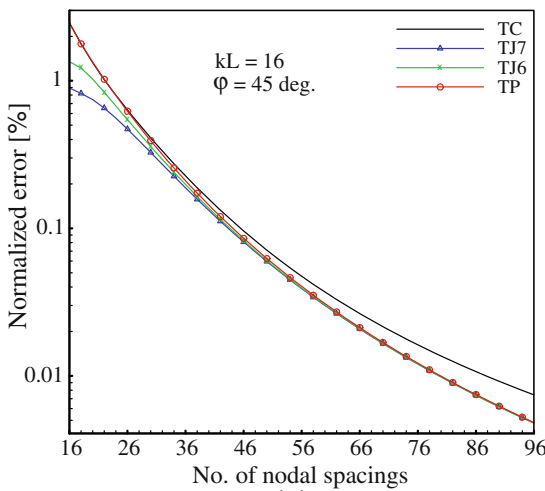

(a)

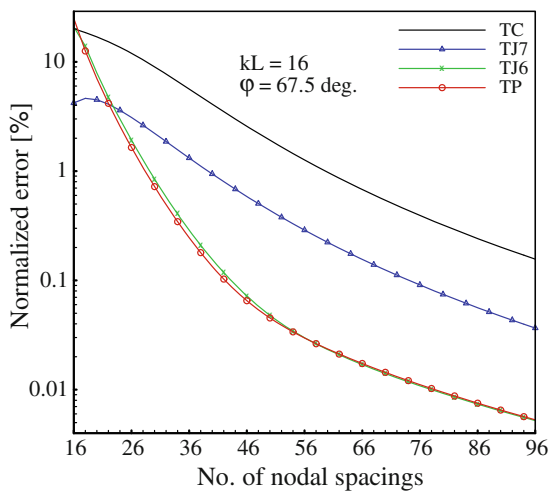

(b)

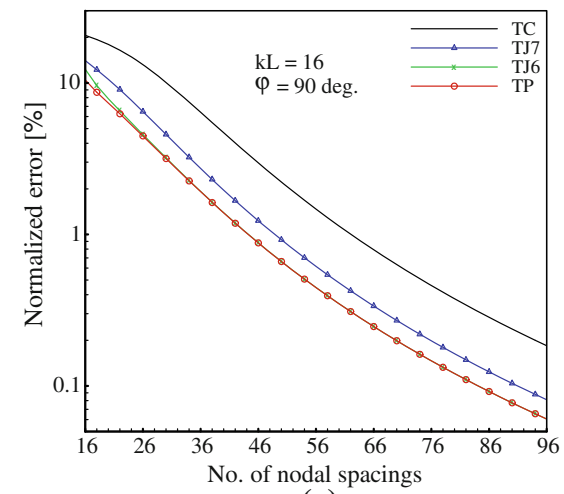

(c)

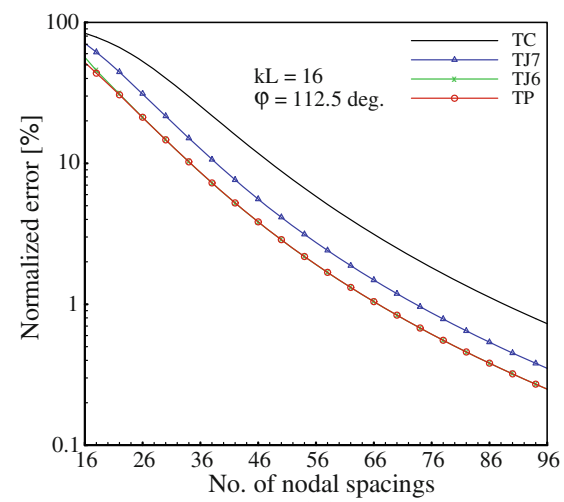

(d)

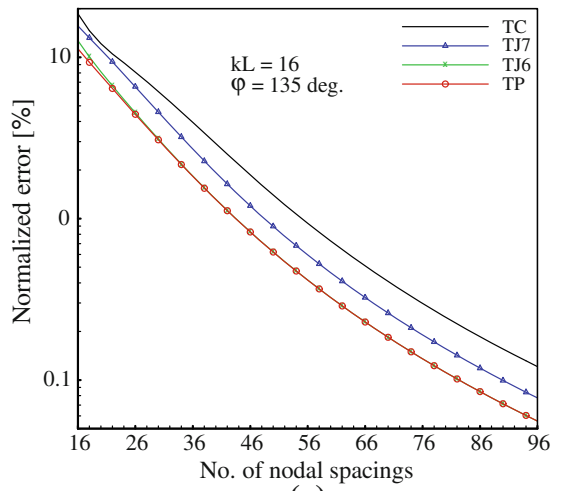

(e)

Fig. 8 Normalized errors of TC, TJ7, TJ6 and TP in the plane-wave problem, see Fig. 4. The number of elements along a domain edge varies from $8\left(N_{n}=2 \pi\right)$ to $48\left(N_{n}=12 \pi\right)$. The wave propagation directions $\varphi$ are $\mathbf{a} 45^{\circ}, \mathbf{b} 67.5^{\circ}, \mathbf{c} 90^{\circ}, \mathbf{d ~} 112.5^{\circ}$ and $\mathbf{e} 135^{\circ}$

Table 4 Average relative errors for results in Fig. 8a-e on the planewave problem

\begin{tabular}{lllll}
\hline$\varphi$ & TC & TP & TJ7 & TJ6 \\
\hline $45^{\circ}$ & 1 & 0.84 & 0.75 & 0.79 \\
$67.5^{\circ}$ & 1 & 0.10 & 0.26 & 0.10 \\
$90^{\circ}$ & 1 & 0.33 & 0.45 & 0.33 \\
$112.5^{\circ}$ & 1 & 0.36 & 0.52 & 0.36 \\
$135^{\circ}$ & 1 & 0.48 & 0.69 & 0.48 \\
\hline
\end{tabular}

Figure 5a,b shows the errors of $\mathrm{TC}$ and $\mathrm{TC}^{*}$ versus $\varphi$ at $5^{\circ}$-interval for $k L=4,8,12$ and 16. $N_{n}$ is fixed at $3 \pi$ by employing the $3 \times 3,6 \times 6,9 \times 9$ and $12 \times 12$ meshes, respectively. TC is consistently more accurate than $\mathrm{TC}^{*}$. Figure $6 a, b$ compares the errors of TP1, TP2 and TP. TP which employs equispaced directions for its plane-wave modes is markedly more accurate than TP1 and TP2. In Fig. 6b, the ordinate is truncated at $50 \%$ for clarity. The largest error is $92.3 \%$ which is produced by TP1 at $k L=$ 16 and $\varphi=107.5^{\circ}$. Figure 7a,b compares TC, TJ7, TJ6 and TP. In Fig. $7 b$, the ordinate is truncated at $50 \%$ for clarity.

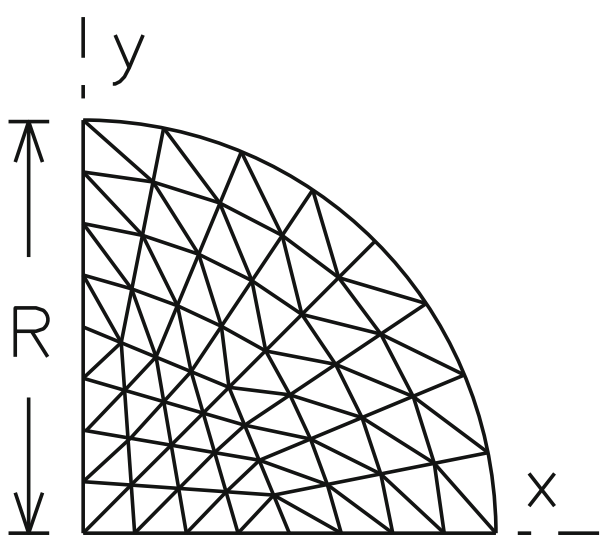

Fig. 9 The circular panel problem in which R equals unity. In the figure, there are $N_{e}(=8)$ elements along each coordinate axis. The exact solution is $u=J_{2}(k r) \cos 2 \theta$. All boundaries are prescribed with the natural boundary condition

The largest error is $73.9 \%$ which is produced by TC at $k L=$ 16 and $\varphi=105^{\circ}$. TC is also the worst model. The best models are TP and TJ6 which yield very close predictions. TJ7 is between TJ6 and TP. For each of the aforementioned figures, 


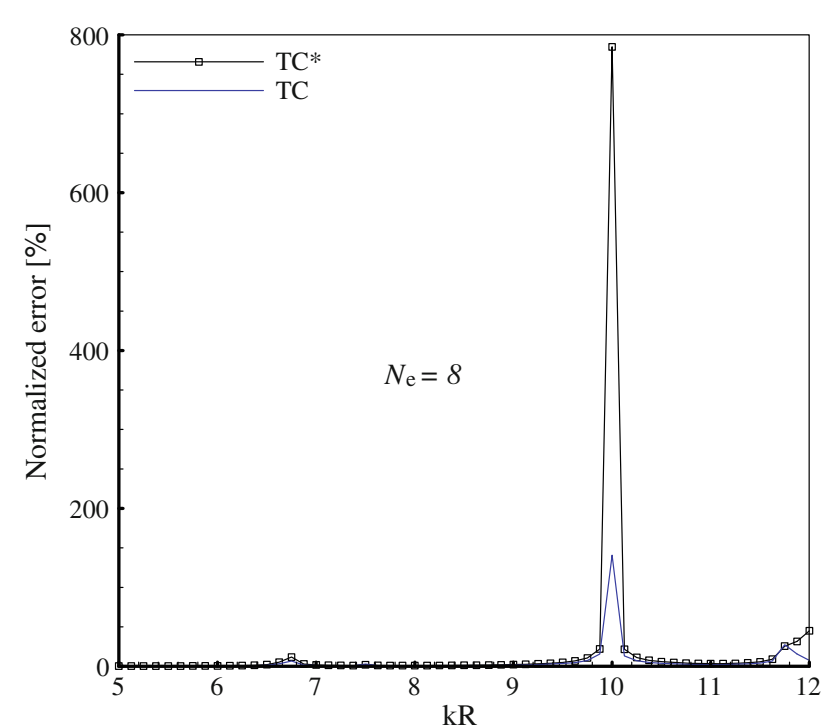

(a)

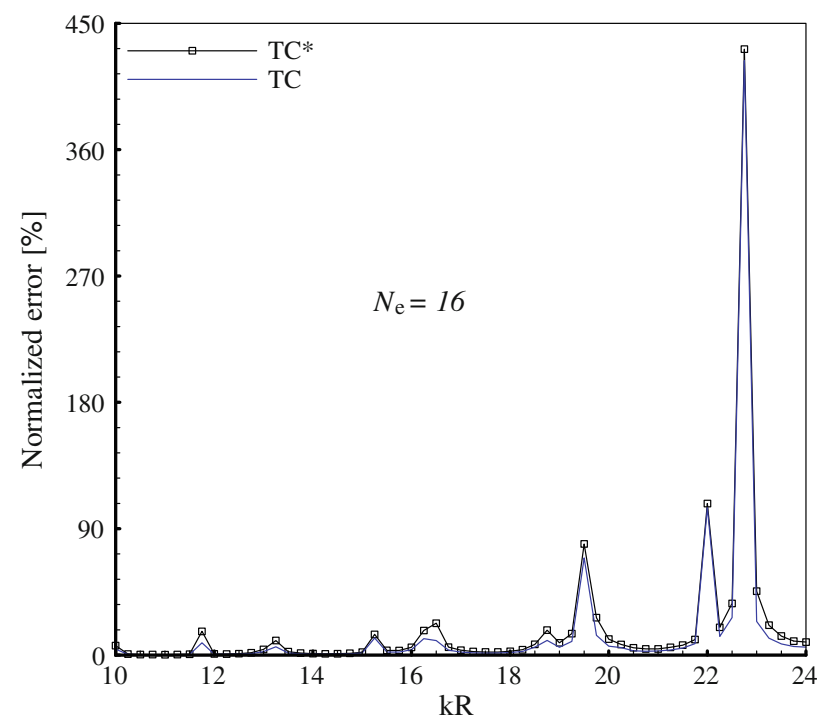

(b)

Fig. 10 Normalized errors of TC* (integrated by the three-point rule) and TC (integrated by the six-point rule) in the circular panel problem, see Fig. 9. In $\mathbf{a}$ and $\mathbf{b}$, there are eight and sixteen elements along each coordinate axis, respectively

the average relative errors with the following definition:

Average relative error

$$
\begin{aligned}
& =\frac{1}{n_{p}} \sum_{i=1}^{n_{p}} \frac{\text { error of the element at the } \mathrm{i} \text {-th } \varphi}{\text { error of TC at the } \mathrm{i} \text {-th } \varphi} \\
& =\frac{1}{n_{p}} \sum_{i=1}^{n_{p}} \frac{\text { normalized error of the element at the } \mathrm{i} \text {-th } \varphi}{\text { normalized error of } \mathrm{TC} \text { at the } \mathrm{i} \text {-th } \varphi}
\end{aligned}
$$

are computed and listed in Table 3. In the equation, $n_{p}$ denotes the number of data points in the concerned figure.

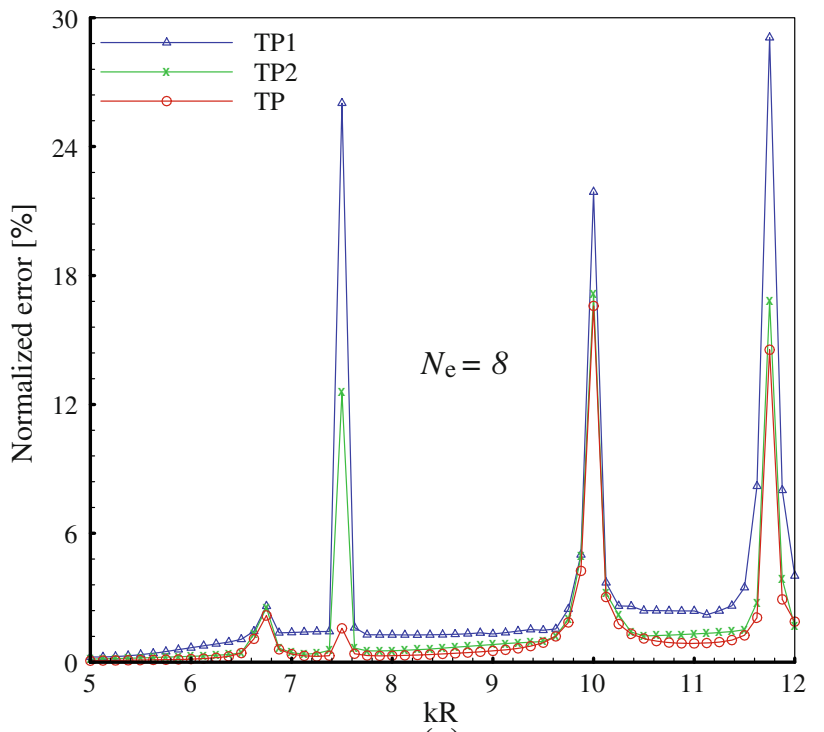

(a)

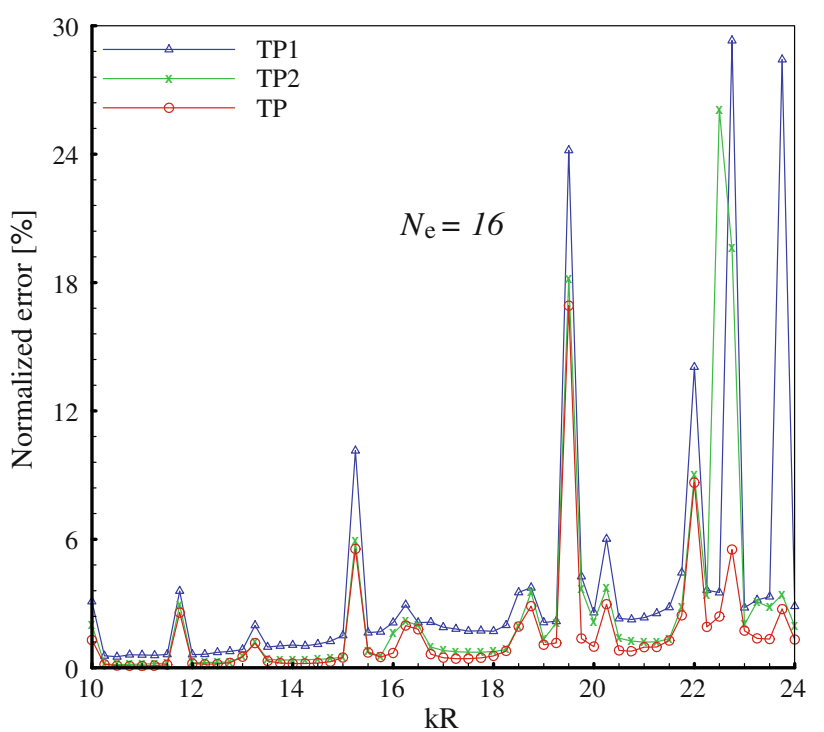

(b)

Fig. 11 Normalized errors of the elements based on the plane-wave modes in the circular panel problem, see Fig. 9. In $\mathbf{a}$ and $\mathbf{b}$, there are eight and sixteen elements along each coordinate axis, respectively

It can be seen from the table that the average relative errors for TP and TJ6 vary from 0.33 to 0.55 . The average relative errors of TP1 and TP2 sometimes exceed one. These values illustrate the importance on choosing the directions of the plane-wave modes. Though TC* is least expensive, it is not advised due to its poor accuracy.

By fixing $k L=16$, the number of nodal spacings per domain edge is varied from $16\left(N_{n}=2 \pi\right)$ to $96\left(N_{n}=12 \pi\right)$ for convergence studies. Figure 8a,b shows the normalized errors of TC, TJ7, TP and TJ6 in semi-log plot for $\varphi=45,67.5,90,112.5$ and $135^{\circ}$. The errors are the smallest at $\varphi=45^{\circ}$. This is understandable as $\varphi=45^{\circ}$ coincident 


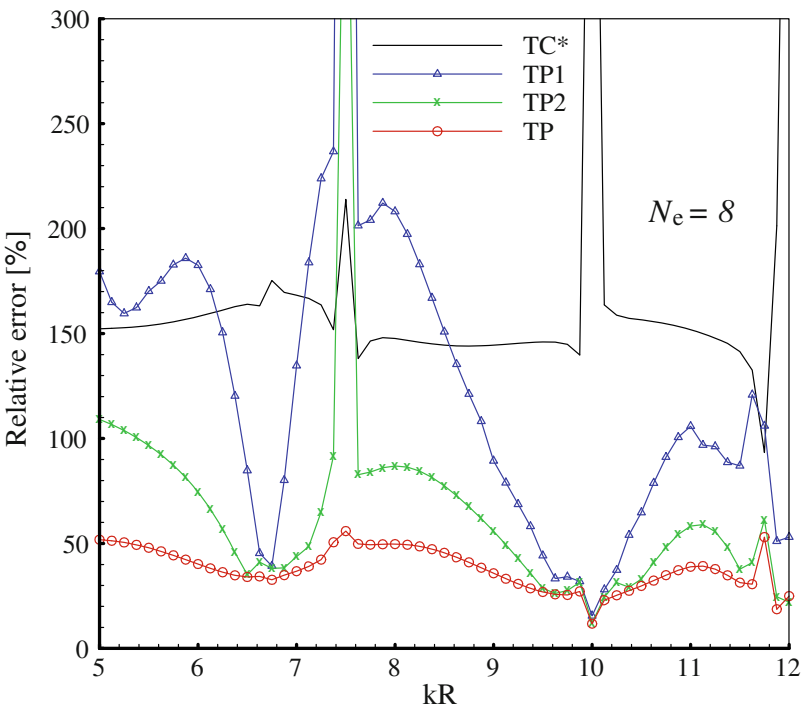

(a)

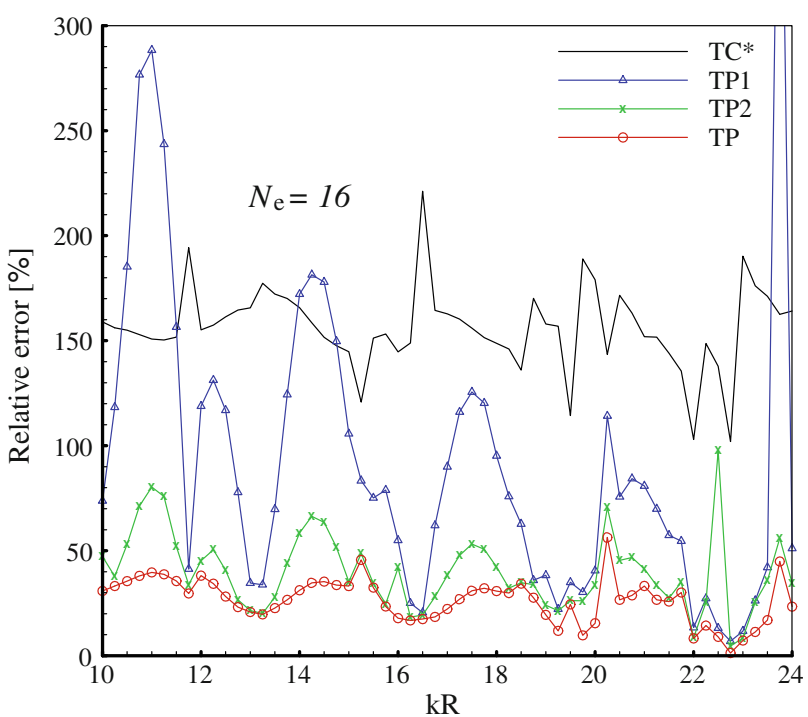

(b)

Fig. 12 Relative errors of TC*, TP1, TP2 and TP in the circular panel problem, see Fig. 9. In a and $\mathbf{b}$, there are eight and sixteen elements along each coordinate axis, respectively

with the direction in which the nodal spacing within the elements is highest. It can also be noted that the convergence rate of all elements are essentially the same. This behaviour is expected as the rate should be controlled by the nodal interpolation order. From Table 4, the average relative errors of TP and TJ6 vary from 0.10 to 0.84 with $4 / 5$ of the values falling below 0.5 .

\subsection{Circular panel problem}

Figure 9 shows a quarter of a circular panel of unit radius modeled by $N_{e}(=8)$ elements along each coordinate axis.

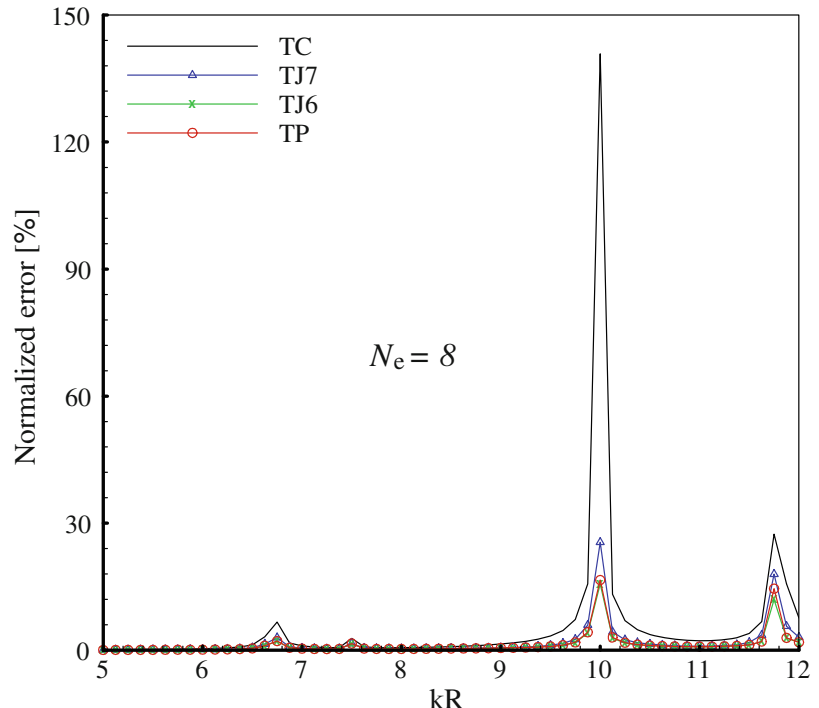

(a)

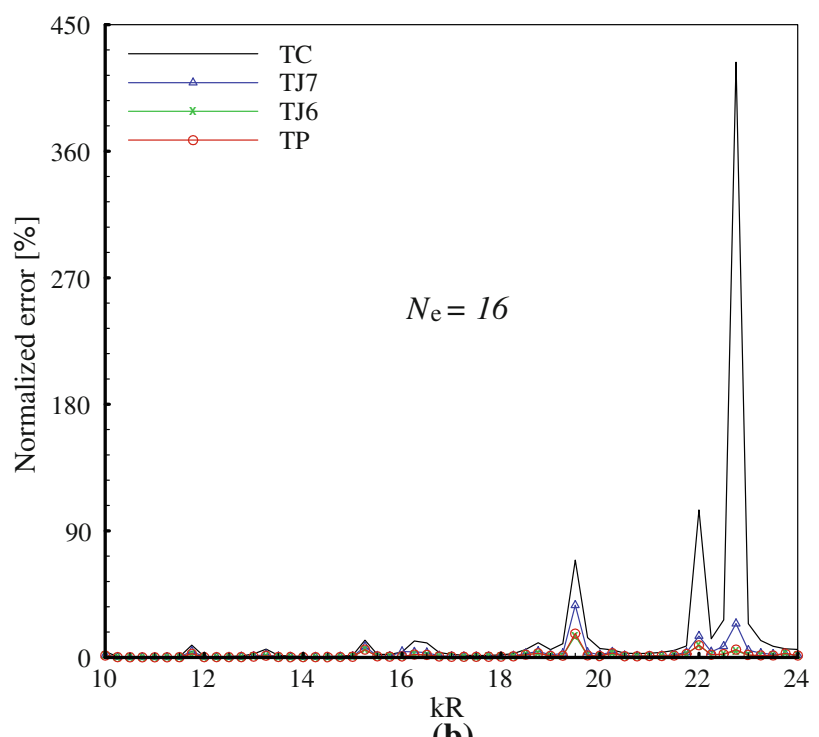

(b)

Fig. 13 Normalized errors of TC, TJ7, TJ6 and TP in the circular panel problem, see Fig. 9. In $\mathbf{a}$ and $\mathbf{b}$, there are eight and sixteen elements along each coordinate axis, respectively

The exact solution of this problem is:

$u_{\text {exact }}=J_{2}(k r) \cos 2 \theta$

Natural boundary condition is prescribed along the entire boundary of the panel. In this example, the relative errors are computed for $5 \leq k R \leq 24$ by using $N_{e}=8$ and $N_{e}=16$. The combinations of $k R$ and $N_{e}$ yield $8.4 \leq N_{n} \leq 20.1$. Figure 10a,b compares the normalized errors of TC and TC* for $N_{e}=8$ and 16, respectively. Figure 11a,b compares the normalized errors of the elements based on the plane-wave modes for $N_{e}=8$ and 16, respectively. The data in Figs. 10 and 11 cluster together. For better clarity, the relative errors (see (41)) of TC*, TP1, TP2 and TP are plotted in Fig. 12a,b 


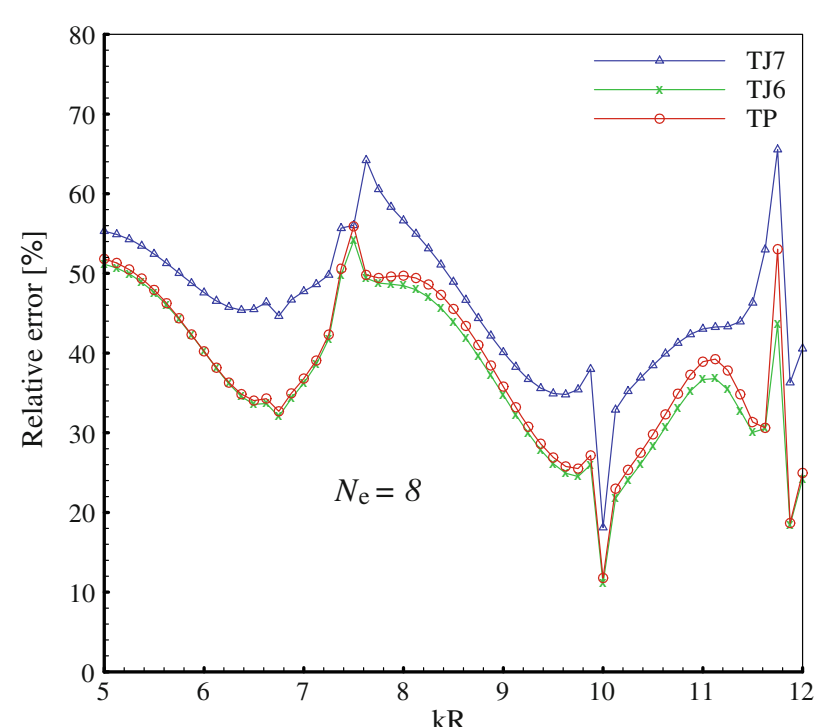

(a)

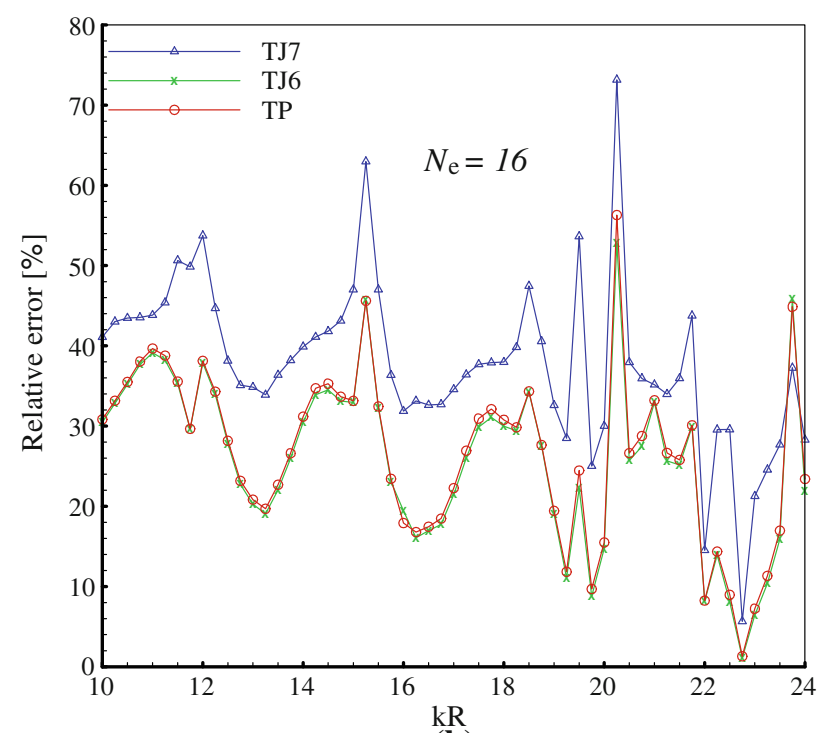

(b)

Fig. 14 Relative errors of TC, TJ7, TJ6 and TP in the circular panel problem, see Fig. 9. In $\mathbf{a}$ and $\mathbf{b}$, there are eight and sixteen elements along each coordinate axis, respectively

for $N_{e}=8$ and 16, respectively. Among the compared elements, $\mathrm{TP}$ is most accurate.

Figure 13a,b compares the normalized errors of TC, TP as well as TJ6 and TJ7 which are based on Bessel solution modes. Besides a few "spines" at which TC is obviously the poorest one in accuracy, most of the data are graphically indistinguishable. For better clarity, the relative errors of TP, TJ6 and TJ7 are plotted in Fig. 14a,b for $N_{e}=8$ and 16, respectively. TP and TJ6 are close and most accurate. TJ7 is less accurate than TP and TJ6. However, it is still consistently more accurate than TC. Table 5 lists the average relative errors, see (44), of the compared elements. The average relative errors of TP and TJ6 vary from 0.26 to 0.38 .
Table 5 Average relative errors for results in Figs. 10a to 11b, 13a, and $13 \mathrm{~b}$ on the circular panel problem

\begin{tabular}{lllllllll}
\hline$N_{e}$ & $k R$ & $\mathrm{TC}^{*}$ & $\mathrm{TC}$ & $\mathrm{TP} 1$ & $\mathrm{TP} 2$ & $\mathrm{TP}$ & $\mathrm{TJ} 7$ & $\mathrm{TJ} 6$ \\
\hline 8 & $5-12$ & 1.66 & 1 & 1.22 & 0.61 & 0.38 & 0.46 & 0.37 \\
16 & $10-24$ & 1.56 & 1 & 0.87 & 0.45 & 0.27 & 0.39 & 0.26 \\
\hline
\end{tabular}

\section{Closure}

Six-node hybrid-Trefftz triangular finite element models with independently assumed boundary and domain modes are devised. The boundary modes are obtained by the standard polynomial-based nodal interpolation and the domain modes are truncated from either the plane-wave or the Bessel solutions. Full attention is exercised in selecting the domain modes such that rank sufficiency and invariance of the elements are secured. The domain modes can be condensed at the element level and, hence, the devised models can readily be incorporated into the standard finite element program framework. Since the boundary modes are obtained by nodal interpolation, the boundary condition can be prescribed in the way identical to that of the continuous Galerkin element. Among the models employing the plane-wave modes, their errors can be marked difference. In this light, the choice of plane-wave modes is important to the element accurate and TP (the element model with equispaced plane-wave propagation directions) is most accurate. Models with six and seven Bessel solution modes are also devised. Similar to our previous experience on quadrilateral models [31], TJ6 (the one with six or the minimal number of domain modes) often yields better accuracy. TP and TJ6 are close in accuracy. In absolute majority of the predictions, the error ratios of TP/TJ6 to TC (the continuous Galerkin element) are less than half. In some cases, the error ratios even drop to one-tenth. Furthermore, when the element geometry is subparametric, the H- and G-matrices of TP (see (22)) can be evaluated by analytical integration as in the plane-wave basis elements $[19,21,38,39]$.

Regarding computational cost, the proposed elements possess 6 nodal dofs. Though the domain modes can be condensed at the element level, the cpu time for forming the element matrix should be higher than the continuous Galerkin model. However, computational costs for large problems are dominated by the solution time and the time for forming the element matrix becomes diminishingly small in proportion. One can note the saving in the number of nodes and thus computational cost for the same accuracy by looking at the convergence studies presented in, e.g., Fig. 8.

Acknowledgments The present work is supported by the Hong Kong Research Grant Council GRF Grant HKU 7167 08E. The authors are grateful to Professor Clark Kimberling for pointing out that the points $\mathrm{C}$ in Figure 3(d) and 3(e) are commonly known as Fermat points (also 
known as Torricelli points and the 1st isogonic centers) of the triangles formed by the corner and side nodes, respectively [42].

Open Access This article is distributed under the terms of the Creative Commons Attribution Noncommercial License which permits any noncommercial use, distribution, and reproduction in any medium, provided the original author(s) and source are credited.

\section{References}

1. Pian THH (1964) Derivation of element stiffness matrices by assumed stress distribution. J AIAA 2:1332-1336

2. Tong P, Pian THH, Lasry SL (1973) A hybrid-element approach to crack problems in plane elasticity. Int J Numer Methods Eng 7:297-308

3. Atluri SN, Kobayashi AS, Nakagaki M (1975) An assumed displacement hybrid finite element method for fracture mechanics. Int J Fract 11:257-271

4. Pian THH (1995) State-of-the-art development of hybrid/mixed finite element method. Finite Elem Anal Des 21:5-20

5. Sze KY, Chan WK, Pian THH (2002) An eight-node hybrid-stress solid-shell element for geometric nonlinear analysis of elastic shells. Int J Numer Methods Eng 55:853-878

6. Jirousek J (1978) Basis for development of large finite elements locally satisfying all field equations. Comput Methods Appl Mech Eng 14:65-92

7. Jirousek J, Zieliński AP (1997) Survey of Trefftz-type element formulations. Comput Struct 63:225-242

8. Freitas JAT, Almeida JP, Pereira EMB (1999) Non-conventional formulations for the finite element method. Comput Mech 23:488501

9. Qin QH (2003) Solving anti-plane problems of piezoelectric materials by the Trefftz finite element approach. Comput Mech 31:461468

10. Wang HT, Sze KY, Yang XM (2006) Analysis of electromechanical stress singularity in piezoelectrics by computed eigensolutions and hybrid-trefftz finite element models. Comput Mech 38:551564

11. Zielinski AP, Herrera I (1987) Trefftz method: fitting boundary conditions. Int J Numer Methods Eng 24:871-891

12. Cheung YK, Jin WG, Zienkiewicz OC (1991) Solution of Helmholtz equation by Trefftz method. Int J Numer Methods Eng 32:6378

13. Herrera I (2000) Trefftz method: a general theory. Numer Methods Partial Differ Equ 16:561-580

14. Sladek J, Sladek V, Keer RV (2002) Global and local Trefftz boundary integral formulations for sound vibration. Adv Eng Softw 33:469-476

15. Chang JR, Liu RF (2004) An asymmetric indirect Trefftz method for solving free-vibration problems. J Sound Vib 275:991-1008

16. Pluymers B, Van Hal B, Vandepitte D, Desmet W (2007) Trefftzbased methods for time-harmonic acoustics. Arch Comput Methods Eng 14:343-381

17. Melenk JM, Babuška I (1997) The partition of unity method. Int J Numer Methods Eng 40:727-758

18. Mayer P, Mandel J (1997) The finite ray element method for the Helmholtz equation of scattering: first numerical experiments. Report 111, Center of Computational Mathematics, University of Colorado at Denver

19. Laghrouche O, Bettess $P$ (2000) Short wave modelling using special finite elements. J Comput Acoust 8:189-210

20. Ortiz P, Sanchez E (2001) An improved partition of unity finite element method for diffraction problems. Int J Numer Methods Eng 50:2727-2740

21. Bettess P, Shirron J, Laghrouche O, Peseux B, Sugimoto R, Trevelyan J (2003) A numerical integration scheme for special finite elements for the Helmholtz equation. Int J Numer Methods Eng $56: 531-552$

22. Farhat C, Harari I, Hetmaniuk U (2003) The discontinuous enrichment method for multiscale analysis. Comput Methods Appl Mech Eng 192:1389-1419

23. Grosu E, Harari I (2008) Studies of the discontinuous enrichment method for two-dimensional acoustics. Finite Elem Anal Des 44:272-287

24. Freitas JAT, Cismaşiu C (2003) Hybrid-Trefftz displacement element for spectral analysis of bounded and unbounded media. Int $\mathrm{J}$ Solids Struct 40:671-699

25. Farhat C, Harari I, Hetmaniuk U (2003) A discontinuous Galerkin method with Lagrange multipliers for the solution of Helmholtz problems in the mid-frequency regime. Comput Methods Appl Mech Eng 192:3195-3209

26. Farhat C, Tezaur R, Wiedemann-Goiran (2004) Higher-order extensions of a discontinuous Galerkin method for mid-frequency Helmholtz problems. Int J Numer Methods Eng 61:1938-1956

27. Tezaur R, Farhat C (2006) Three-dimensional discontinuous Galerkin elements with plane waves and Lagrange multipliers for the solution of mid-frequency Helmholtz problems. Int J Numer Methods Eng 66:796-815

28. Stojek M (1998) Least square Trefftz-type elements for the Helmholtz equation. Int J Numer Methods Eng 41:831-849

29. Stojek M, Markiewicz M, Mahrenholtz O (2000) Diffraction loads on multiple vertical cylinders with rectangular cross section by Trefftz-type finite elements. Comput Struct 75:335-345

30. Sze KY, Cheung YK (2008) A hybrid-Trefftz finite element model for Helmholtz problem. Commun Numer Methods Eng 24:20472060

31. Sze KY, Liu GH, Fan H (2010) Four- and eight-node hybrid-Trefftz quadrilateral finite element models for Helmholtz problem. Comput Methods Appl Mech Eng 199:598-614

32. Desmet W, Van Hal B, Sas P, Vandepitte D (2002) A computationally efficient prediction technique for the steady-state dynamic analysis of coupled vibro-acoustic systems. Adv Eng Softw 33:527-540

33. Pluymers B, Desmet W, Vandepitte D, Sas P (2004) Application of an efficient wave based prediction technique for the anlaysis of vibro-acoustic radiation problems. J Comput Appl Math 168:353364

34. Van Hal B, Desmet W, Vandepitte D (2005) Hybrid finite elementwave based method for steady-state interior structural-acoustic problems. Comput Struct 83:167-180

35. Spilker RL, Maskeri SM, Kania E (1981) Plane isoparametric hybrid-stress elements: invariance and optimal sampling. Int $\mathrm{J}$ Numer Methods Eng 17:1469-1496

36. Sze KY, Chow CL, Chen WJ (1992) On invariance of isoparametric hybrid elements. Commun Numer Meth Eng 8:385-406

37. Brezzi F, Fortin M (1997) Mixed and hybrid finite element methods. Springer in computational mathematics, vol 15. Springer-Verlag, New York

38. Deraemaeker A, Babuška I, Bouillard P (1999) Dispersion and pollution of the fem solution for the Helmholtz equation in one, two and three dimensions. Int J Numer Methods Eng 46:471-499

39. Gabard G (1992) Exact integration of polynomial-exponential products with application to wave-based numerical methods. Commun Numer Methods Eng 25:237-246

40. Herrera I, Sabina FJ (1978) Connectivity as an alternative to boundary integral equations: construction of bases. Proc Nat Acad Sci USA 75(5):2059-2069

41. Cook RD, Malkus DS, Plesha ME (1989) Concepts and applications of finite element analysis, 3rd edn. Wiley, New York

42. Kimberling C (1998) Triangle centers and central triangles. Congr Numer 129:1-295 